\title{
Interférences
}

Ars scribendi

5 | 2009

Historia / Persona

\section{La persona oratoire entre rhétorique, biographie et histoire}

Le cas des Controuersiae de Sénèque le Rhéteur

\section{Charles Guérin}

\section{OpenEdition}

\section{Journals}

Édition électronique

URL : http://journals.openedition.org/interferences/897

DOI : 10.4000/interferences.897

ISSN : 1777-5485

\section{Éditeur}

HiSoMA - Histoire et sources des Mondes antiques

Édition imprimée

Date de publication : 1 janvier 2009

ISSN : 1777-5485

\section{Référence électronique}

Charles Guérin, «La persona oratoire entre rhétorique, biographie et histoire », Interférences [En ligne],

5 | 2009, mis en ligne le 10 juillet 2014, consulté le 15 septembre 2020. URL : http://

journals.openedition.org/interferences/897 ; DOI : https://doi.org/10.4000/interferences.897

Ce document a été généré automatiquement le 15 septembre 2020.

Tous droits réservés 


\title{
La persona oratoire entre rhétorique, biographie et histoire
}

\author{
Le cas des Controuersiae de Sénèque le Rhéteur
}

\author{
Charles Guérin
}

\section{Introduction}

1 Rédigées au début du règne de Caligula ${ }^{1}$, les Controuersiae de Sénèque le Rhéteur occupent une place mal définie dans la tradition rhétorique, tant par leur objet que par leur mode de structuration ou leur contenu. L'ouvrage entend recenser les traits (sententiae), les divisions (diuisiones, la manière de traiter une question) et les couleurs (colores, l'orientation donnée à l'argumentation et à la narration ${ }^{2}$ ) de rhéteurs ayant pratiqué la déclamation entre la fin de la République et les débuts du Principat ${ }^{3}$. Le texte, qui est aujourd'hui passablement amputé, était à l'origine divisé en dix livres organisés par sujets de déclamation ${ }^{4}$. Chaque chapitre de ces différents livres partait d'une loi fictive, énonçait un cas d'espèce, puis recensait le traitement que différents rhéteurs en avaient proposé. En Contr. I, 5, la loi de départ précise par exemple que quiconque surprend un adultère peut tuer les amants sans dommage. Un soldat héroïque, qui a perdu les deux mains dans une bataille, surprend sa femme en train de fauter et demande à son fils d'infliger à sa place la punition prévue en mettant à mort les amants. Le fils ayant refusé, son père le déshérite. Les rhéteurs vont s'affronter sur cette cause fictive et Sénèque nous présentera les traits employés, les différentes divisions de la cause et les couleurs mises en œuvre par chaque déclamateur pour l'une et l'autre partie.

Chacun de ces livres s'ouvre sur une préface, qui présente en détail un ou plusieurs des rhéteurs dont les déclamations sont par la suite citées ${ }^{5}$. Ces préfaces, qui recensent, dans leur état de mutilation actuel, dix-huit rhéteurs, apportent à leur sujet des renseignements biographiques - caractéristiques morales et physiques, origine, situation sociale, circonstances de la mort - ainsi que des informations techniques qualités et défauts oratoires, méthode de travail, compétences intellectuelles générales. 
Par ces préfaces, les Controuersiae fournissent une galerie de portraits d'orateurs et de rhéteurs extrêmement riche, mais le font de manière indirecte, puisque l'organisation générale de l'ouvrage place ces portraits à la périphérie et non au centre d'un propos organisé suivant les thèmes de déclamation et non suivant les déclamateurs. Néanmoins, parce que chaque citation de trait, de couleur ou de division est accompagnée du nom du rhéteur qui en est l'auteur ${ }^{6}$, le texte se présente bien comme un témoignage sur les caractéristiques particulières à chaque déclamateur.

3 Cette structuration complexe, et souvent déroutante, amène à soulever une question fondamentale, qui est celle des outils intellectuels utilisés par l'auteur pour faire le portrait de ces différents rhéteurs et, partant, pour rendre compte, organiser et rendre intelligibles les caractéristiques de chacun d'entre eux. Dans le foisonnement de son texte, le projet de Sénèque le Rhéteur est bien de faire apprécier au lecteur ce qui faisait la spécificité oratoire ou déclamatoire de ces personnages, leurs qualités et leurs défauts, et ainsi de faire comprendre ce qu'était l'idiosyncrasie de chacun de ces praticiens, soit la somme des traits stylistiques et comportementaux qui constituaient leur persona ${ }^{7}$. Toute la difficulté consiste alors à comprendre quels schémas intellectuels se trouvent mis en œuvre par l'auteur dans une œuvre explicitement située au point de rencontre de l'histoire, de la biographie et de la rhétorique.

4 La tradition critique a souvent tenté d'éliminer la question en insistant sur l'absence de structure et de doctrine rhétoriques clairement identifiables dans les écrits de Sénèque le Rhéteur. L. Sussman souligne ainsi que Sénèque le Rhéteur ne fait pas usage du vocabulaire technique traditionnel ${ }^{8}$ et, plus encore, qu'il n'offre à son lecteur que de très rares développements proprement théoriques ${ }^{9}$. Mettant ainsi en doute les capacités théoriques et la rigueur méthodique de leur auteur, L. Sussman fait des Controuersiae et des Suasoriae des textes de critique littéraire à portée biographique, sans rapport véritable avec la technique rhétorique elle-même. Cette approche se retrouve très largement dans la monographie de J. Fairweather qui, comme L. Sussman, souligne l'absence d'un lexique rhétorique explicitement structuré dans l'ouvrage et insiste sur la non-systématicité du propos de Sénèque ${ }^{10}$. Pour ces deux auteurs, les Controuersiae et les Suasoriae n'offriraient aucune structuration identifiable ${ }^{11}$ et se détacheraient entièrement de la tradition rhétorique antérieure. Florilège biographique et critique, le propos de Sénèque ne s'appuierait sur aucun système, et devrait être lu comme un assemblage de remarques qui ne serait pas unifié par des méthodes cohérentes d'analyse.

5 Une telle approche suppose, chez L. Sussman comme chez J. Fairweather, une présentation de la rhétorique antérieure comme une tradition rigide et dogmatique, qu'il est alors aisé d'opposer à la souplesse et à la fluidité de la critique caractéristique de Sénèque le Rhéteur. Chez ces deux auteurs, la tradition rhétorique est en effet appréhendée comme un ensemble homogène de règles prescriptives, énoncées dans des manuels et transmises de façon répétitive. Disparaissent ainsi de la réflexion d'autres modes de formalisation rhétorique tout aussi présents dans la tradition, en particulier ceux propres à la rhétorique descriptive représentée par le Brutus de Cicéron ${ }^{12}$, texte que L. Sussman réduit à un «mechanical and dogmatic criticism ${ }^{13}$ " et dont $\mathrm{J}$. Fairweather passe sous silence la spécificité dans la tradition ${ }^{14}$. Supposer une rupture totale entre les traditions antérieures et les textes de Sénèque permet alors de présenter l'auteur des Controuersiae et des Suasoriae comme un critique "intuitif» s'appuyant uniquement sur son expérience et son goût. Il n'est dès lors plus 
véritablement nécessaire de s'interroger sur la possible existence d'une méthode ou d'une structuration théorique qui viendrait donner sens à la galerie de portraits bâtie par Sénèque.

6 Cette approche entraîne avec elle de nombreuses difficultés méthodologiques, et pousse à lire les ouvrages de Sénèque d'une manière qui n'est pas totalement satisfaisante. Elle incite tout d'abord le lecteur à rapprocher ces textes d'une tradition biographique avec laquelle ils s'accordent mal, sauf à négliger entièrement leur portée didactique ${ }^{15}$. Elle évacue ensuite une tradition rhétorique tout à fait nécessaire à la compréhension du texte : décrire les spécificités d'un orateur - ou d'un déclamateur est une démarche rhétorique tout à fait reconnue, et l'on se souvient que Cicéron, dès le De oratore, ne se contente pas d'énoncer des règles, mais porte également une attention scrupuleuse à ce qui fait la particularité des orateurs qu'il mentionne. Considérer Sénèque le Rhéteur comme un hapax ou, plus exactement, comme le primus inuentor d'un protocole descriptif - de surcroît non thématisé - représente une forme d'abandon herméneutique, qui se ressent d'ailleurs dans les lectures le plus souvent proposées. Ainsi J. Fairweather choisit-elle, conformément à ses remarques de départ, de déconstruire totalement le texte de Sénèque pour le réorganiser, puis l'analyser, selon les schémas «traditionnels » de la rhétorique prescriptive (celle des officia oratoris) afin de comparer le contenu des Controuersiae et des Suasoriae aux doctrines de Cicéron et de Quintilien ${ }^{16}$. Sénèque étant supposé ne pas avoir de dessein théorique propre, il n'est en effet plus nécessaire de chercher à le déceler. L'originalité proclamée de son texte est alors ramenée à des structures connues, dont rien ne garantit pourtant la valeur explicative puisque l'on a auparavant affirmé qu'elles appartenaient à des traditions différentes.

7 Il n'est certes pas question de faire de Sénèque le théoricien qu'il n'est en aucune manière. Mais il ne paraît pas pour autant illégitime de s'interroger sur la nature de ses écrits et sur la manière dont il donne sens aux portraits d'orateurs qu'il nous présente, en cherchant à découvrir quelle influence la pensée rhétorique a pu exercer sur les descriptions contenues dans ses préfaces. Car si ces portraits, comme le remarque J. Fairweather, ne sont pas structurés conformément aux divisions rhétoriques les plus courantes ${ }^{17}$, la portée didactique du texte, sur laquelle nous aurons à revenir, suppose l'existence d'un appareil évaluatif permettant à Sénèque le Rhéteur de critiquer ou de louer les différents déclamateurs qu'il nous présente. Tout comme la prétention sénéquienne à la désinvolture et au décousu dissimule en réalité une construction du texte extrêmement solide et raffinée ${ }^{18}$, la présentation apparemment lâche des différents déclamateurs s'appuie sur des structures théoriques sous-jacentes qu'il convient d'autant plus de déceler qu'elles ne sont pas explicitement évoquées par l'auteur. On ne tentera donc pas de repérer une théorie en forme dans ces développements ${ }^{19}$, mais simplement de comprendre quels modes de pensée rhétoriques sous-tendent des descriptions dont on ne peut supposer qu'elles sont faites au hasard et sans la moindre cohérence. On pourra alors montrer que le texte des Controuersiae s'inscrit certes dans les traditions historique et biographique, comme le veulent L. Sussman et J. Fairweather, mais qu'il repose également sur une appréhension proprement rhétorique des personnages qu'il dépeint. En donnant un sens nouveau à la tradition qu'il reprend, Sénèque parvient en effet à faire se rencontrer préoccupations rhétoriques, biographiques et historiques d'une façon plus maitrisée et théorisée qu'on ne veut habituellement le reconnaître. Ainsi, même si l'on entend lire les préfaces des 
Controuersiae comme des textes biographiques, ces textes biographiques n'en demeurent pas moins structurés au moyen d'une pensée proprement rhétorique.

L'objet de cet article est donc d'analyser les structures rhétoriques d'interprétation qui sont mises en place par Sénèque le Rhéteur pour rendre intelligibles les caractéristiques des déclamateurs et présenter leurs personae respectives dans les préfaces des Controuersiae. Nous nous interrogerons tout d'abord sur le rapport général qu'entretient le texte à l'histoire, à la biographie et à la rhétorique, pour examiner ensuite la manière dont Sénèque le Rhéteur modifie les outils d'analyse propres à la rhétorique descriptive dont il est l'héritier afin de les adapter à son objet et de leur donner une valeur historique nouvelle.

\section{Histoire, biographie et rhétorique : quel modèle pour la rédaction des Controuersiae?}

\section{Sénèque le Rhéteur historien et antiquaire?}

9 Si l'ambiguïté des Controuersiae apparaît immédiatement, tant il est difficile de faire le départ entre histoire, biographie et appréciations proprement rhétoriques au sein du texte, il est néanmoins évident que Sénèque le Rhéteur ne prétend jamais faire œuvre d'historien. Son projet n'est pas similaire à celui du Brutus, où Cicéron annonçait qu'il présenterait tous les orateurs de Rome en les situant dans une chronologie clairement établie et en les identifiant de façon précise avant de les caractériser ${ }^{20}$. Que l'objectivité de ces premières intentions soit rapidement mise à mal et que le texte cicéronien prenne rapidement un tour plus évaluatif que strictement descriptif importe peu : le cadre demeure, tout au long du traité, celui d'un ouvrage historique, dont le principe avait été inspiré par le Liber annalis d'Atticus mentionné en Brut. 11-15 et $19^{21}$. Sénèque le Rhéteur, pour sa part, affirme d'emblée le caractère subjectif et évaluatif de son entreprise sans jamais prétendre ni à l'objectivité, ni à l'exhaustivité normalement attendues d'une narration historique ${ }^{22}$. Explicitement énoncé, son projet consiste simplement à répondre à une demande émise par ses fils ${ }^{23}$ et à formuler un jugement sur les différents rhéteurs qu'il lui avait été donné d'entendre :

... iubetis enim quid de his declamatoribus sentiam qui in aetatem meam inciderunt indicare, et si qua memoriae meae nondum elapsa sunt ab illis dicta colligere, ut, quamuis notitiae uestrae subducti sint, tamen non credatis tantum de illis sed et iudicetis.

... vous voulez que je vous expose mon opinion sur les déclamateurs dont j'ai été le contemporain, et que je rassemble en un recueil celles de leurs paroles qui ne se sont pas encore enfuies de ma mémoire; de la sorte, bien que vous n'ayez pu les connaître directement, vous vous formerez vous-mêmes une opinion sur eux au lieu de vous borner à la recevoir (Sen. Rhet., Contr. I. praef. 1, trad. H. Bornecque modifiée).

Sous cet abord naiff, le projet est plus complexe qu'il n'y paraît. La perspective est en premier lieu didactique. Sénèque le Rhéteur entend en effet donner à ses fils - et à ses lecteurs - le moyen de former correctement leur jugement en leur fournissant un ensemble d'exemples organisés qui leur permette d'affiner leur goût :

Omnia autem genera corruptarum quoque sententiarum de industria pono, quia facilius et quid imitandum et quid uitandum sit docemur exemplo.

C'est à dessein que je cite toutes les espèces de traits défectueux, parce qu'un exemple nous enseigne plus facilement ce qu'il faut imiter et ce qu'il faut éviter (Sen. Rhet., Contr. IX, 2, 27, trad. H. Bornecque modifiée). 
11 L'auteur entend ainsi offrir un catalogue de modèles et de contre-modèles qui rendront possible une imitation maîtrisée. Reprenant les jugements formulés par certains déclamateurs ou exprimant directement ses préférences et ses critiques, Sénèque adjoint à sa description une démarche proprement évaluative et pédagogique. Ses préférences sont clairement exprimées, au point que Sénèque rassemble ses déclamateurs favoris dans un primum tetradeum sur lequel il entend imposer son jugement. Ses fils peuvent bien apprécier à leur gré les autres déclamateurs, au moyen des exemples que leur père leur a fournis et qui leur ont donné les références leur permettant de formuler un jugement éclairé, mais Latron, Fuscus, Albucius et Gallion sont classés à part. Leur talent ne saurait être mis en question :

Primum tetradeum quod faciam quaeritis? Latronis, Fusci, Albuci, Gallionis. Hi quotiens conflixissent, penes Latronem gloria fuisset, penes Gallionem palma; reliquos ut uobis uidebitur conponite : ego uobis omnium feci potestatem. Hos minus nobiles sinite in partem abire, Paternum et Moderatum, Fabium et si quis est nec clari nominis nec ignoti.

Vous vous demandez qui je fais entrer dans le premier quadrige? C'est Latron, Fuscus, Albucius, Gallion : toutes les fois qu'ils auraient fait assaut d'éloquence, la gloire eût été pour Latron et la palme pour Gallion. Les autres, rangez-les dans l'ordre qui vous plaît, je vous ai mis à même de le faire. Ceux qui sont moins illustres, laissez-les dormir en paix, les Paternus, les Moderatus, les Fabius, et tous ceux qui ne sont ni connus, ni inconnus (Sen. Rhet., Contr.X praef.13, trad. H. Bornecque).

12 La dimension prescriptive du texte apparaît alors dans toute son évidence, alors même que le fil de la chronologie tend à disparaître : car ce qui rapproche ces déclamateurs n'est pas une communauté d'influence, ou l'appartenance à une même école, mais la simple appréciation de Sénèque tendant à valoriser les modèles qu'il apprécie. En lieu et place d'une histoire des pratiques, se substitue donc un projet évaluatif permettant à l'auteur de transmettre une opinion dont il estime qu'elle est fondée sur une réflexion approfondie ${ }^{24}$. On ne s'étonne plus, dès lors, que Sénèque laisse délibérément de côté certains orateurs jugés médiocres (Lucius Magius et Lucius Asprenus par exemple, en Contr. X praef. 2) ou qu'il critique ouvertement l'engouement que manifestent ses fils pour des orateurs qui n'en sont pas dignes ${ }^{25}$. Le projet historique, qui imposait l'exhaustivité à Cicéron dans son Brutus ${ }^{26}$, s'efface ici pour laisser place à la subjectivité affirmée de l'auteur.

Didactique, axiologique et partiale, la démarche adoptée est également mémorielle, mais débouche sur un texte qui n'est, paradoxalement, jamais conçu comme une œuvre d'historien. Sénèque le Rhéteur présente en effet son ouvrage comme le moyen de transmettre aux générations futures le souvenir de rhéteurs menacés par l'oubli :

Ipsis quoque multum praestaturus uideor, quibus obliuio inminet nisi aliquid quo memoria eorum producatur posteris tradetur. Fere enim aut nulli commentarii maximorum declamatorum extant aut, quod peius est, falsi. Itaque ne aut ignoti sint aut aliter quam debent noti, summa cum fide suum cuique reddam.

À ces orateurs mêmes, je rendrai, je crois, un grand service; l'oubli les menace, si l'on ne transmet pas à la postérité quelque chose qui prolonge leur souvenir. En effet, il n'existe pour ainsi dire pas de recueil de notes prises en écoutant les plus grands déclamateurs, ou ceux qui existent sont apocryphes, ce qui est pire. Aussi, pour éviter qu'ils ne demeurent ignorés ou qu'ils ne soient connus autrement qu'ils ne le devraient, je rendrai à chacun son dû avec une scrupuleuse exactitude. (Sen. Rhet., Contr. I praef. 11, trad. H. Bornecque modifiée).

14 Cette transmission est fondée sur une autopsie revendiquée à de multiples reprises, et sur des souvenirs personnels abondants que l'auteur a conservés grâce à une mémoire 
qu'il nous présente comme prodigieuse ${ }^{27}$. C'est sur cette mémoire que repose l'ensemble de l'ouvrage, puisque les déclamateurs dont il est question n'ont pour la plupart pas laissé de notes (comme on l'a vu dans l'exemple précédent) ou que leurs ouvrages ont parfois été détruits ${ }^{28}$. Ce faisant, Sénèque le Rhéteur entend rétablir certaines vérités, en particulier à propos de son ami Latron, injustement critiqué d'après lui ${ }^{29}$. Il pourra également confondre les plagiaires qui réutilisent des traits ou des couleurs qu'ils ont subtilisés à des déclamateurs oubliés ${ }^{30}$.

Ainsi, la position auctoriale de Sénèque le Rhéteur rend difficile de voir dans son œuvre le résultat d'un quelconque projet historique. La dimension didactique du propos, tout d'abord, tend à donner à l'ouvrage une valeur technique. De plus, Sénèque présente luimême son texte comme le résultat d'un vagabondage dans ses souvenirs, qui n'est contraint par aucun ordre: les sententiae d'un même orateur, ou celles prononcées à une même occasion, ne seront pas regroupées, mais abordées selon les hasards de la mémoire. Il faut certes faire la part, dans ce désordre apparent, à la convention littéraire : L. Sussman a bien montré que, en filigrane de cette désinvolture, se dessinait une architecture précisément construite ${ }^{31}$. Mais cette architecture ne repose ni sur une chronologie, ni sur un effort de synthèse autre que celui imposé par les thèmes déclamatoires recensés dans chaque livre. Tout au plus peut-on remarquer que les déclamateurs dépeints dans les préfaces voient leurs sententiae et leurs colores davantage représentés dans le livre qui suit ${ }^{32}$. Sénèque le Rhéteur ne s'engage donc en aucun cas dans un travail d'historien, mais, en endossant le rôle d'un antiquaire, entend exposer à son gré ses propres souvenirs d'une période dont il a été le témoin privilégié ${ }^{33}$ :

Illud necesse est inpetrem, ne me quasi certum aliquem ordinem uelitis sequi in contrahendis quae mihi occurrent; necesse est enim per omnia studia mea errem et passim quidquid obuenerit adprehendam.

Mais je dois vous demander de ne pas exiger que je suive un ordre défini en rassemblant les souvenirs qui vont se présenter à moi ; il est nécessaire que j'aille au hasard à travers toutes mes années d'études et que je saisisse au passage tout ce qui me reviendra en mémoire (Sen. Rhet., Contr. I praef. 4, trad. H. Bornecque modifiée).

De fait, dans la mise en scène propre au récit, ce sont parfois les demandes des enfants qui orientent le propos de l'auteur et lui font aborder telle ou telle figure oratoire, comme dans le cas de Votienus Montanus, au début du Livre IX :

Vltro Votieni Montani mentionem intulistis; et uelim uos subinde aliqua nomina mihi offerre, quibus euocetur memoria mea, quae quomodo senilis per se marcet, admonita et aliquando lacessita facile se colliget.

C'est vous qui avez fait mention de Votienus Montanus, et je serais heureux que, de temps à autre, vous me fournissiez quelques noms qui excitent ma mémoire, car si, déjà vieille, elle est naturellement engourdie, pourtant, quand on la réveille et qu'on l'aiguillonne un peu, elle retrouve facilement son ancienne vigueur (Sen. Rhet., Contr. IX praef. 1, trad. H. Bornecque modifiée).

On est donc loin d'un parcours gouverné par un ordre chronologiquement ou thématiquement fixé : antiquaire et technicien, Sénèque construit un ouvrage qui ne prétend pas appartenir au genre historique. 


\section{Sénèque le Rhéteur biographe et critique rhétorique?}

Sénèque le Rhéteur entraîne avec elle deux conséquences quant à la manière qu'a l'auteur d'aborder les caractéristiques de chaque déclamateur. La dimension mémorielle du texte impose tout d'abord à Sénèque de conserver et de transmettre des détails qu'il est seul à connaître. Le propos dépasse donc immédiatement la simple description de la pratique déclamatoire proprement dite pour entrer dans le domaine de la biographie. Sénèque recense ainsi des informations qui relèvent des topiques normales de l'éloge, mais également de la uita, comme la méthodologie employée par les déclamateurs, leur apparence physique, leurs caractéristiques sociales, morales et psychologiques et, enfin, les circonstances de leur mort. Ces éléments sont parfois corrélés à la pratique oratoire. Un physique qui avantage, une mémoire exceptionnelle ou défectueuse, un trait de caractère particulier représentent autant de facteurs explicatifs des succès ou des échecs rencontrés par ces déclamateurs. On apprend ainsi que Cassius Seuerus était grandement avantagé par sa prestance corporelle ou que le manque de fiducia sui dont souffrait Albucius rendait son style instable ${ }^{34}$. Le lien entre notation biographique et projet critique apparait alors de manière évidente. Mais ces éléments biographiques sont parfois mentionnés de manière totalement indépendante, fournissant alors des caractérisations qui se situent hors du champ d'intérêt défini par le projet de départ. Ainsi, Sénèque le Rhéteur nous renseigne dûment sur la duplicité morale de Labienus ${ }^{35}$, mais cette information n'éclaire en rien sa pratique oratoire, qui n'est jamais abordée en termes de moralité ou d'immoralité. Dans les passages de ce type, l'unité même de l'ouvrage peut être mise en question.

Que les indications biographiques fournies par Sénèque présentent ou non une valeur critique, qu'elles soient corrélées à la pratique déclamatoire ou détachées d'elle, jamais elles ne se trouvent inscrites dans une structure récurrente ou sérielle comme l'exigerait un projet biographique véritable ${ }^{36}$. Ainsi, les six catégories que nous avons pu recenser ne constituent pas un levier d'analyse ou un élément structurant, dans la mesure où elles ne sont pas exploitées de façon systématique pour chaque orateur et où l'auteur ne les utilise pas pour élaborer des grilles de comparaison. Seuls les traits saillants ou inhabituels retiennent l'attention de Sénèque le Rhéteur, qui ne développe jamais une structure de présentation fixe. Anecdotique plus que strictement biographique, le texte de Sénèque n'est pas consciemment élaboré à partir des modèles qui régissent habituellement la rédaction des uitae ${ }^{37}$.

Au delà de cette tendance biographique et anecdotique, la seconde orientation de l'ouvrage de Sénèque, la description et l'évaluation des traits stylistiques, pousse à rapprocher les Controuersiae du modèle de la rhétorique descriptive tel qu'il est mis en œuvre par le Brutus de Cicéron. Rédigé en 46 av. J.-C., le Brutus prend la suite du projet rhétorique développé par Cicéron dans le De oratore et s'intéresse aux pratiques réelles des orateurs de Rome. S'éloignant du domaine de la norme rhétorique entendue au sens strict, Cicéron expose, tout au long de cet ouvrage unique dans la tradition, les variations qui affectent, selon les époques et les individus, la mise en pratique de cette norme. Il entend ainsi rendre compte de l'évolution de la pratique oratoire à Rome en recensant toutes les figures d'orateurs dont la Cité avait pu garder le souvenir. L'organisation adoptée par Cicéron dans ce texte reposait sur deux structures d'analyse. La première était chronologique, puisque le texte abordait ces orateurs par 
génération en les regroupant autour d'une grande figure qui, en quelque sorte, servait de diapason stylistique à son époque (aetas ${ }^{38}$ ). La seconde était technique et stylistique, cette structure chronologique se doublant d'une opposition binaire, présente à chaque époque, entre deux types d'orateurs, l'un ample et l'autre restreint. L'idiosyncrasie de chacun pouvait donc s'analyser en référence à ces deux grilles : l'une fondée sur la stabilité et la transmission de modèles stylistiques liés à une époque et à un environnement donnés, l'autre fondée sur une opposition de modèles fixes dépassant les évolutions historiques. Dans les deux cas, la diversité des pratiques se trouve ramenée à des genres d'orateurs (oratorum genera ${ }^{39}$ ) qui reposent sur des critères de constitution différents. Le premier genus, chronologique, est donc fondé sur une similitude stylistique des orateurs, similitude analysée en termes de proximité temporelle. Le personnage de Brutus résume bien la méthode adoptée par Cicéron :

Ego uero, inquit Brutus, et delector ista quasi notatione temporum et ad id quod instituisti, oratorum genera distinguere aetatibus, istam diligentiam esse accommodatam puto.

En ce qui me concerne, dit Brutus, d'abord je prends plaisir à cette espèce de chronologie, et puis j'estime que pour l'objet que tu t'es proposé, qui est de différencier des types d'orateurs en fonction de leur époque, ce souci d'exactitude est excellent (Cic., Brut. 74, trad. J. Martha modifiée).

Le second genus repose quant à lui sur la construction systématique de paires d'orateurs, dont on oppose terme à terme les comportements oratoires, l'éloquence simple et restreinte d'une part, l'éloquence ample de l'autre ${ }^{40}$. Les différents orateurs se trouvant subsumés sous deux grandes structures stables, le propos de Cicéron peut dépasser le simple enregistrement de traits individuels et utiliser ces deux genera historique et stylistique pour structurer son histoire de l'éloquence romaine. Néanmoins, le genre ample étant systématiquement valorisé dans la deuxième structure $^{41}$, la description historique et stylistique se mue rapidement en appareil critique et implicitement prescriptif.

Bien que Sénèque n'entende pas faire œuvre d'historien et qu'il ne laisse pas apparaître de structuration évidente dans les portraits qu'il propose, il convient de s'interroger précisément sur la possible présence des modes de structuration propres au Brutus dans les Controuersiae. Car en proposant des modèles et des contre-modèles (quid imitandum est? quid uitandum est?; Contr. IX, 2, 27), en valorisant ou en critiquant certains comportements oratoires, Sénèque se détache lui aussi, comme on l'a vu, d'une approche descriptive pour adopter une démarche critique. Il laisse ainsi entrevoir l'existence, dans son propos, d'un appareil évaluatif qui motive une approche consciente des préférences déclamatoires qu'il expose. On constatera alors que, si l'architecture théorique du Brutus n'est pas directement transposable dans le texte qui nous occupe, l'idée d'une caractérisation à la fois chronologique et générique sous-tend bel et bien l'ouvrage de Sénèque.

\section{La diversité oratoire et l'impossible structuration chronologique}

Bien que son texte ne puisse pas, comme on l'a vu, être intégré à la tradition de l' historia, Sénèque le Rhéteur n'en fait pas moins intervenir dans son propos des modes de pensée historiques et prête une véritable attention à la modification progressive des pratiques oratoires et déclamatoires. Ainsi, la déclamation se trouve abordée comme un 
genre évolutif, et la préface du livre I présente des réflexions sur le statut des controverses et leur transformation: en passant de la thesis à la controuersia, la déclamation de causes fictives a en effet pris un visage nouveau qui ne s'est imposé que récemment ${ }^{42}$. Sénèque le Rhéteur note également l'introduction de nouvelles manières de faire ${ }^{43}$, marque un intérêt véritable pour l'évolution des modes d'enseignement ${ }^{44}$ et traite de rhéteurs qui appartiennent à trois générations successives ${ }^{45}$. Enfin, le texte sur lequel s'ouvre les Controuersiae, la préface du livre I, adopte une perspective résolument historique qui tranche avec la tournure antiquaire et anecdotique qui prévaut dans le reste du texte : l'auteur y replace l'ensemble de son projet dans le cadre plus large de l'évolution des pratiques oratoires de la fin de la République jusqu'au règne de Caligula, évolution qu'il conçoit explicitement comme une décadence continue $^{46}$.

24 Cette approche historicisée de la pratique devrait permettre la formalisation d'une notion de genus oratoire conçue de façon chronologique, proche de celle employée dans le Brutus, où ce genus oratoire permettait de réduire la diversité des orateurs en les ramenant chacun à un modèle défini et identifiable en termes de génération. Le projet consistant à formaliser une identité oratoire propre à chaque époque est certes absent des textes de Sénèque le Rhéteur. Cette absence ne lui interdit pourtant pas d'aborder les caractéristiques oratoires en termes de nouveauté ou d'ancienneté, et de proposer ainsi une structuration des individualités oratoires fondée sur une forme de chronologie stylistique. L'orateur Titus Labienus ${ }^{47}$, présenté au livre $\mathrm{X}$ des Controuersiae, va représenter une sorte de figure pivot qui fournit une grille de lecture possible des évolutions stylistiques enregistrées par Sénèque :

Adfectabat enim censorium supercilium, cum alius animo esset : magnus orator, qui multa impedimenta eluctatus ad famam ingeni confitentibus magis hominibus peruenerat quam uolentibus. [...] Color orationis antiquae, uigor nouae, cultus inter nostrum ac prius saeculum medius, ut illum posset utraque pars sibi uindicare.

Il affectait en effet de prendre le front sévère d'un censeur, quoiqu'il eût un caractère bien différent: c'était un grand orateur qui, à travers mille difficultés, était arrivé à une réputation de talent, de l'aveu forcé plutôt que du consentement de ses contemporains. [...] Il unissait la couleur de l'ancien style à la vigueur du nouveau; les ornements qu'il utilisait tenaient de notre siècle et du dernier, en sorte que les deux époques pouvaient le revendiquer au même titre (Sen. Rhet., Contr. X praef. 4-5, trad. H. Bornecque modifiée).

Labienus possède en effet un color que Sénèque rattache à un modèle stylistique ancien (oratio antiqua), un uigor caractéristique d'un modèle nouveau (noua oratio) et un travail stylistique intermédiaire, situé à égale distance de ces deux modèles. Le problème est que le contenu définitoire de ce color, de ce uigor et de ce cultus n'est jamais exposé qu'en termes relatifs. Jamais, dans l'ouvrage, Sénèque ne prendra la peine de préciser les caractéristiques des colores anciens et nouveaux, ou de nous faire voir quels aspects du travail stylistique (cultus) sont désormais favorisés par les «modernes ». Il ne reste plus au lecteur qu'à le déduire du reste de l'ouvrage, puisque ces réalités stylistiques ne seront jamais véritablement thématisées.

Pourtant, la figure de Labienus permet bien de matérialiser le passage d'un modèle ancien à un modèle nouveau, et laisse deviner une réflexion portant sur les raisons de cette transformation qui va au delà de la simple déploration face à la décadence des pratiques oratoires et déclamatoires. La tradition de la rhétorique descriptive a deux manières de penser la transformation des caractéristiques stylistiques d'une génération d'orateur à une autre, et donc de différencier les comportements oratoires propres à 
chaque époque. La première est sociale et politique. Il s'agit, dans ce cas, de prendre en compte l'influence des conditions d'exercice de l'éloquence sur les formes que prend cette dernière. Dans le cadre des Controuersiae, la méthode consisterait à rapprocher d'une part le passage du régime républicain au principat et, d'autre part, le développement du cultus, le changement du color et l'accroissement du uigor, selon une analyse qui sera celle du personnage de Maternus, dans le Dialogus de Tacite, lorsqu'il insiste sur le lien qui unit la forme de l'éloquence et les conditions dans lesquelles elle s'exerce ${ }^{48}$. Mais aucune réflexion de ce type n'apparaitra jamais chez Sénèque, celui-ci se concentrant sur le second aspect possible de l'analyse des modèles et de leur succession, celui, strictement esthétique et non plus social et politique, de l'imitation, selon une approche qui, cette fois-ci, sera celle du personnage d'Aper dans le texte de Tacite ${ }^{49}$, et qui correspond également à la méthode descriptive principalement employée par Cicéron. Dès 55 av. J.-C., dans le De oratore, Cicéron mettait en place un modèle d'analyse visant à expliquer le caractère stylistique de chaque époque à partir d'un orateur qui servirait de modèle à une génération :

Quid enim causae censetis esse cur aetates extulerint singulae singula prope genera dicendi [

...]. Quorum quamdiu mansit imitatio, tam diu genus illud dicendi studiumque uixit...

À quelle cause attribuez-vous, en effet, que chaque période, ou peu s'en faut, ait produit un genre particulier d'éloquence? Aussi longtemps qu'ils trouvèrent des imitateurs, le style et le goût de ces orateurs se maintinrent (Cic., De orat. II, 92-95, trad. E. Courbaud modifiée).

Il s'agit là de la première formulation d'une analyse qui sera ensuite développée dans le Brutus, à travers la structuration chronologique des orateurs romains autour d'une grande figure donnant le «ton" stylistique de son époque. Dans le cas de Sénèque le Rhéteur, l'utilisation de cette grille d'interprétation ne permettra pas d'expliquer le passage du modèle ancien au modèle nouveau, mais figera l'analyse dans le modèle nouveau dont l'auteur détaille la diffusion et l'enracinement.

En bonne tradition cicéronienne, Sénèque le Rhéteur place en effet l'imitation au cœur $\mathrm{du}$ processus de formation des orateurs ${ }^{50}$. Cicéron préconisait le choix d'un modèle unique, qu'il fallait sélectionner avec soin pour qu'il corresponde aux caractéristiques physiques et intellectuelles de l'apprenti orateur. Ce choix dérivait d'une réflexion sur le convenable et l'adaptation à soi. Chacun doit en effet s'orienter dans le choix de son modèle en prenant en compte sa propre nature. Ainsi Antoine pousse-t-il Sulpicius à calquer son comportement oratoire sur celui de Crassus, car ce modèle conviendra, d'après lui, parfaitement à ses qualités :

Vidi statim indolem neque dimisi tempus eum sum cohortatus, ut forum sibi ludum putaret esse ad discendum, magistrum autem, quem uellet, eligeret; me quidem si audiret, L. Crassum. [...] Omnino in illud genus eum Crassi magnificum atque praeclarum natura ipsa ducebat, sed ea non satis proficere potuisset, nisi eodem studio atque imitatione intendisset atque ita dicere consuesset, ut tota mente Crassum atque omni animo intueretur. Je vis d'emblée les qualités qui étaient les siennes, et, sans perdre de temps, je l'exhortai à considérer le forum comme son école et son lieu d'apprentissage, et à choisir le maître qu'il voudrait: s'il voulait m'en croire, il choisirait L. Crassus [...]. Sa propre nature le portait tout à fait à ce genre brillant et éclatant qui était celui de Crassus, mais celle-ci n'aurait pu se développer suffisamment s'il ne s'était, avec la même application, à la fois exercé par l'imitation et habitué à parler en ayant constamment tout son esprit et toute sa pensée tournés vers Crassus (Cic., De orat. II, 89). utile à ses fils précisément parce qu'il leur permettra de se détacher des modèles qui 
leur sont contemporains et qui, selon l'irrésistible mouvement de décadence exposé dans la préface du livre I des Controuersiae, sont inférieurs à ceux qui prévalaient auparavant, une époque dépravée ne pouvant fournir que des modèles qui le sont tout autant :

Facitis autem, iuuenes mei, rem necessariam et utilem quod non contenti exemplis saeculi uestri prioris quoque uultis cognoscere. Primum quia, quo plura exempla inspecta sunt, plus in eloquentiam proficitur. [...] Deinde ut possitis aestimare in quantum cotidie ingenia decrescant et nescio qua iniquitate naturae eloquentia se retro tulerit...

Vous faites d'ailleurs, mes enfants, une chose nécessaire et utile en ne vous contentant pas des modèles de votre époque en voulant connaître également ceux de la précédente; d'abord, plus on a de modèles sous les yeux, plus on fait de progrès vers l'éloquence. [...] Ensuite, vous pourrez peser dans quelle mesure les talents décroissent chaque jour, et, par je ne sais quelle défaveur de la nature, l'éloquence a rétrogradé... (Sen. Rhet., Contr. I praef. 6, trad. H. Bornecque).

Il leur permettra également d'avoir sous les yeux des sources d'imitation diverses qui garantiront le meilleur apprentissage possible ${ }^{51}$ :

Non est unus, quamuis praecipuus sit, imitandus, quia numquam par fit imitator auctori.

Il ne faut pas imiter un modèle unique, si éminent soit-il, car l'imitateur n'arrive jamais au niveau de son modèle (Sen. Rhet., Contr. I praef. 6).

Ce faisant, Sénèque le Rhéteur fournit un schéma général d'interprétation qui pourrait permettre d'expliquer l'état de la pratique oratoire contemporaine, d'éclairer les différences qui distinguent l'ancien et le nouveau style et de décrire de façon fine les caractéristiques de chacun.

Sénèque perçoit en effet l'imitation comme une inévitable dégradation, ce qu'elle n'était en aucun cas aux yeux de Cicéron. Par conséquent, toute continuation d'un modèle aboutit nécessairement à son affaiblissement ${ }^{52}$, affaiblissement que l'on cherchera à compenser par une diversification des modèles ${ }^{53}$, la pluralité des sources d'inspiration permettant de masquer la dégradation d'une qualité donnée par la présence d'une autre. Le travail d'imitation n'est donc pas perçu comme une intégration à soi-même des particularités propres à un autre, mais comme une simple reproduction, largement superficielle. Ainsi s'explique la récurrence de notations où Sénèque nous décrit des efforts d'imitation erratiques car soumis au goût du moment, critiquant par exemple l'incapacité d'Albucius à se tenir à un modèle défini :

Hoc illi accedebat inconstantia iudicii : quem proxime dicentem commode audierat imitari uolebat. Memini omnibus illum omissis rebus apud Fabianum philosophum, tanto iuueniorem quam ipse erat, cum codicibus sedere; memini admiratione Hermagorae stupentem ad imitationem eius ardescere. Nulla erat fiducia ingenii sui, et ideo adsidua mutatio; itaque dum genera dicendi transfert et modo exilis esse uolt nudisque rebus haerere, modo horridus et ualens potius quam cultus, modo breuis et concinnus, modo nimis se attollit, modo nimis se deprimit, ingenio suo inlusit et longe deterius senex dixit quam iuuenis dixerat...

À cela s'ajoutait l'inconstance de son goût: le dernier orateur qu'il venait d'entendre bien parler, il voulait toujours l'imiter. Je me souviens que, toute affaire cessante, il alla s'asseoir et prendre des notes à l'école du philosophe Fabianus qui était deux fois plus jeune que lui. Je me souviens que, tout béant d'admiration pour Hermagoras, il brûlait de l'imiter. Il n'avait aucune confiance en ses propres moyens : de là ces changements perpétuels ; aussi, en passant d'un style à l'autre, en voulant être tantôt sec et s'en tenir à la simple nudité des choses, tantôt rude et énergique plutôt qu'élégant, tantôt bref et précis, en s'élevant parfois trop haut, en redescendant trop bas, il gâta son talent et parla dans sa vieillesse beaucoup plus 
mal que dans son âge mûr... (Sen. Rhet., Contr. VII praef. 4-5, trad. H. Bornecque modifiée). frappants pour les reproduire de manière caricaturale, ce qui correspond précisément à ce que Cicéron conseillait d'éviter ${ }^{54}$. Un élément capital se trouve laissé de côté par l'analyse de Sénèque, qui tient au changement dans les modes d'imitation qu'implique le passage d'un art oratoire politique et efficace à une pratique déclamatoire et scolastique. Le jeune rhetor n'est plus un jeune orator : il ne part pas, ainsi qu'Antoine le conseillait à Sulpicius, choisir son modèle sur le forum, mais le découvre à l'école. Ceux qui s'offriront à son imitation seront tout d'abord son maître et ses camarades, puis des maîtres concurrents ou des tenants d'autres écoles. Disparaît, bien évidemment, toute la dimension politique qui donnait son sens profond à l'imitation oratoire sous la République ${ }^{55}$. L'imitation se trouve par conséquent limitée à un phénomène d'ordre strictement esthétique, ce qui entraîne plusieurs conséquences.

Cette approche de l'évolution du style uniquement centrée sur la reproduction de traits esthétiques, en dehors de toute implication politique, fait tout d'abord surgir une forme antique de la question du plagiat. Ainsi L. Cestius Pius se plaint-il d'être imité et caricaturé par son élève Argentarius, qui répond en affirmant qu'il régénère un modèle décati :

Quid putatis, aiebat, Argentarium esse? Cesti simius est. Solebat et Graece dicere: $\dot{o}$

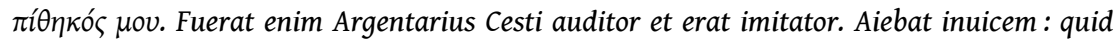
putatis esse Cestium nisi Cesti cinerem? et sic solebat iurare: " per manes praeceptoris mei Cesti ».

«À votre avis, disait-il, qu'est-ce qu'Argentarius ? C'est le singe de Cestius ». Il avait coutume de l'appeler aussi "mon singe", en grec. En effet, Argentarius avait été l'élève de Cestius et il l'imitait. À son tour, Argentarius disait : « À votre avis, qu'estce que Cestius sinon les cendres de Cestius? ? Et il avait coutume de jurer par «les mânes de mon maître Cestius » (Sen. Rhet., Contr. IX, 3, 12, trad. H. Bornecque).

L'imitation ainsi entendue fait naître en second lieu de véritables difficultés dans la pratique. Cestius, le même dont il était question à l'instant, cherche ainsi à imiter des déclamateurs talentueux qui lui sont infiniment supérieurs. Mais, parce qu'il est grec, il ne dispose pas de l'abondance verbale qui lui permettrait de mener à bien les descriptions qu'il calque sur ses modèles. Il reste donc fréquemment paralysé au beau milieu d'un développement, faute de matière :

... itaque, quotiens latius aliquid describere ausus est, totiens substitit, utique cum se ad imitationem magni alicuius ingeni derexerat...

... aussi, toutes les fois qu'il osa se risquer dans une description un peu longue, il resta court, surtout lorsqu'il se proposait d'imiter quelqu'un qui avait du talent... (Sen. Rhet., Contr. VII, 1, 27, trad. H. Bornecque modifiée).

Plus généralement, il semble que l'on aboutisse nécessairement à un échec quand l'infériorité au modèle est trop flagrante, comme dans le cas de Fuluius Sparsus :

Sparsus autem dicebat violenter, sed dure. Ad imitationem se Latronis derexerat, nec tamen umquam similis illi erat, nisi cum eadem diceret. Vtebatur suis verbis, Latronis sententiis. Quant à Sparsus, il parlait avec force mais avec rudesse. Il s'était mis à imiter Latron, et d'ailleurs, ne lui ressemblait jamais, sauf quand il disait exactement la même chose. Il se servait de mots à lui et des pensées de Latron (Sen. Rhet., Contr. X praef. 11, trad. H. Bornecque).

Le regard critique que porte Sénèque le Rhéteur sur le processus d'imitation l'amène donc à n'avoir pour modèle d'analyse que celui du plagiat, de la dégradation par 
rapport au modèle et de la transmission des défauts ${ }^{56}$. Papirius Fabianus en fournit un exemple parlant, qui ne parvient tout d'abord à saisir et à reproduire que les travers de son maître avant de réussir à se détacher totalement de lui :

Exercebatur apud Arellium Fuscum, cuius genus dicendi imitatus plus deinde laboris impendit ut similitudinem eius effugeret quam inpenderat ut exprimeret.

Il s'exerçait sous la direction d'Arellius Fuscus et, après avoir imité son éloquence, il lui fallut, dans la suite, dépenser plus de travail pour fuir cette ressemblance qu'il n'en avait dépensé pour l'acquérir (Sen. Rhet., Contr. II praef. 1, trad. H. Bornecque).

Ce mécanisme d'imitation et de dégradation représente donc un outil d'interprétation qui relève strictement de la critique stylistique mais ne permet pas le développement d'une structuration historique semblable à celles que l'on rencontre dans le Brutus et le Dialogus: manque aux Controuersiae un élargissement de la perspective au delà du rapport direct entre maître et élève, ou modèle et imitateur. On constate ainsi que Sénèque le Rhéteur n'aborde pas la question de la stabilisation du style d'une époque, et qu'il ne propose aucun levier d'analyse efficace sur ce point. La persona des différents rhéteurs ne pourra donc pas être inscrite dans un schéma historique d'évolution et de transmission des comportements oratoires.

\section{Structuration stylistique de l'identité oratoire et innovation critique}

Reste par conséquent à déterminer si Sénèque exploite la seconde forme de structuration possible, la structuration par types d'orateurs que l'on rencontre également dans les développements descriptifs du corpus rhétorique cicéronien. Soit un genus d'orateur qui n'est pas organisé à partir de sa situation dans l'histoire de l'éloquence mais à partir de traits stylistiques clairement identifiés et qui seraient présents à des époques différentes. Deux divisions ont été employées pour caractériser les différents orateurs dans la tradition rhétorique. La plus courante est une division ternaire, et se fonde sur la doctrine théophrastique des $\chi \alpha \rho \alpha \kappa \tau \varepsilon ́ p \varepsilon \varsigma ~ \lambda o ́ \gamma o v^{57}$. Elle apparaît dans la phase ultime de la réflexion rhétorique cicéronienne, dans l'Orator et le De optimo genere. Bien que contestée par Cicéron qui, à la fin de l'année 46 av. J.-C., défend l'idée selon laquelle il n'existerait qu'un seul type d'orateurs, cette division permet de ramener les différents orateurs aux trois niveaux stylistiques déjà recensés dans la Rhétorique à Herennius ${ }^{58}$, le style simple, le style moyen et le style ample :

Oratorum genera esse dicuntur tamquam poetarum [...]. Oratorum autem si quis ita numerat plura genera, ut alios grandis aut grauis aut copiosos, alios tenuis aut subtilis aut breuis, alios eis interiectos et tamquam medios putet, de hominibus dicit aliquid, de re parum.

On dit qu'il y a des "genres d'orateurs", comme il y en a de poètes. [...] Chez les orateurs, si on veut dénombrer plusieurs genres en considérant les uns comme grands ou graves ou abondants, d'autres comme minces ou subtils ou brefs, d'autres comme intermédiaires et tenant le milieu entre eux, ce qu'on dit a un sens en ce qui concerne les individus, mais n'en a guère pour la chose (Cic., De. opt. gen. 1-3, trad. A. Yon).

La seconde division possible est binaire. Elle s'appuie sur une tradition ancienne réactivée par Cicéron, en 46 av.J.-C., qui oppose style simple et style ample, précisément dans le contexte de la querelle atticiste. Il existe ainsi, d'après la doctrine du Brutus, deux types de bons orateurs, l'un restreint, l'autre ample : 
Quoniam ergo oratorum bonorum (hos enim quaerimus) duo genera sunt, unum attenuate presseque, alterum sublate ampleque dicentium, etsi id melius est quod splendidius et magnificentius, tamen in bonis omnia quae summa sunt iure laudantur.

Puisqu'il existe deux genres de bons orateurs (ce sont ceux, en effet, qui nous intéressent), les uns employant un style simple et précis, les autres un style élevé et ample, et bien que le style qui a le plus d'éclat et de magnificence soit le meilleur, on loue à bon droit tout ce qui atteint l'excellence chez ces bons orateurs (Cic., Brut. 201).

41 La structuration ternaire est entièrement absente des Controuersiae. À l'inverse, on relève tout au long du texte la présence d'une opposition binaire entre une éloquence ample et une éloquence restreinte. Un certain nombre d'orateurs se trouvent ainsi rangés dans la catégorie des orateurs secs (aridi) ${ }^{59}$, quand d'autres sont caractérisés par leur ampleur et leur brillant (splendor) ${ }^{60}$. Entièrement implicite, ce mode de caractérisation et d'organisation des différentes figures d'orateurs n'est jamais inscrit dans un appareil précisément organisé puisqu'il ne concerne pas tous les orateurs. Cicéron, dans le Brutus, tentait à l'inverse de généraliser cette catégorisation même si elle n'était explicite que dans certains cas ${ }^{61}$ : au terme de l'analyse cicéronienne, tout orateur pouvait et devait trouver sa place dans cette construction. Plus encore, il apparaît rapidement que cette opposition entre sécheresse et ampleur ne présente pas la stabilité nécessaire permettant de fournir une armature efficace de caractérisation oratoire. Au delà du fait qu'elles ne se trouvent pas explicitement structurées, ces catégories n'opposent pas de grands ensembles comportementaux permettant de formaliser une persona oratoire fixe. Sous une première forme, elles mettent en balance des manières de faire temporaires. Ainsi Arellius Fuscus est-il splendidus dans ses argumentations mais aridus dans le reste de son discours :

Erat explicatio Fusci Arelli splendida quidem sed operosa et implicata [...] principia, argumenta, narrationes aride dicebantur.

Les développements d'Arellius Fuscus étaient brillants, il est vrai, mais pénibles et embrouillés [...] l'exorde, la démonstration et les narrations étaient traités de manière sèche (Sen. Rhet., Contr. II praef. 1, trad. H. Bornecque modifiée).

Dans d'autres cas, la notion employée ne sert pas à caractériser un type, mais simplement à stigmatiser un défaut. Ainsi, le collègue anonyme de Sparsus évoqué au livre X est-il un declamator subtilis sed aridus (Contr. X praef. 11), expression où aridus ne renvoie en aucun cas à une catégorie d'orateurs. Arellius Fuscus, qui vient d'être évoqué, peut lui aussi être ramené à ce cas de figure, puisque son style, tantôt splendidus et tantôt aridus, est intégralement défectueux. Arellius souffre en effet d'une :

summa inaequalitas orationis, quae modo exilis erat, modo nimia licentia uaga et effusa.

son style était extrêmement changeant, tantôt sec, tantôt diffus et sans retenue, sous l'effet d'une liberté excessive (Sen. Rhet., Contr. II praef. 1).

La manière dont Sénèque le Rhéteur aborde les traits distinctifs des orateurs simples ou amples qu'il décrit est donc très éloignée de la méthode que Cicéron avait élaborée. Il ne s'agit plus, en réalité, de différencier deux ou trois types de comportements oratoires également valides, mais d'opposer une manière correcte et une manière défectueuse de parler, comme l'on peut s'en rendre compte en élargissant la perspective au delà de l'opposition entre style simple et style ample. L'exemple de Cassius Seuerus est à cet égard parlant. Ainsi, pour caractériser ce rhéteur, Sénèque semble tout d'abord mettre en place une opposition entre deux types de style : 
Omnia ergo habebat quae illum ut bene declamaret instruerent : phrasin non uulgarem nec sordidam sed electam, genus dicendi non remissum aut languidum sed ardens et concitatum, non lentas nec uacuas explicationes, sed plus sensuum quam uerborum habentes...

Par conséquent, il possédait toutes les qualités nécessaires pour bien déclamer, une diction sans vulgarité ni bassesse, mais soignée, une parole non pas détendue ou languissante mais pleine de feu et d'animation, des développements non pas lents ou vides mais renfermant plus d'idées que de mots (Sen. Rhet., Contr. III praef. 7, trad. H. Bornecque modifiée). le genus dissolutum (style flasque) celui du mediocre genus, le genus aridum et exangue (style sec et sans vigueur) répondant quant à lui à la figura extenuata ${ }^{62}$. C'est l'emploi excessif des caractéristiques normales de chaque style qui conduit à verser dans ces genres défectueux ${ }^{63}$. C'est donc un appareil d'évaluation stylistique complet qui se trouve mis en place dans la Rhétorique à Herennius, où les styles défectueux ne prennent sens et ne s'analysent qu'à partir des genres positivement définis dont ils détournent et forcent les caractéristiques. Cet appareil est précisément ce qui fait défaut dans les Controuersiae: si les caractérisations négatives apparaissent bien chez Sénèque le Rhéteur, les styles positivement définis auxquels ils devraient renvoyer manquent. La caractérisation des orateurs présentés par Sénèque ne peut donc reposer sur la doctrine des vices voisins.

Notre hypothèse est que la manière qu'a Sénèque le Rhéteur de structurer les caractéristiques oratoires en se fondant sur l'opposition entre qualités et défauts stylistiques nous fait sortir de tous les schémas descriptifs habituels pour exploiter un autre appareil rhétorique : la doctrine des vertus oratoires, qui n'a jamais été employée de la sorte avant notre auteur dans la tradition latine. Les vertus oratoires se trouvent normalement réparties en deux structures. Un premier ensemble théorique est formé 


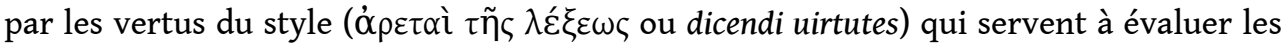
orateurs et non à les caractériser. Le style doit ainsi respecter, en plus de la correction lexicale et syntaxique, trois exigences pour être considéré comme correct: la clarté, l'ornement et la propriété, c'est-à-dire l'adaptation au sujet, à l'auditeur, à l'orateur luimême et aux circonstances. Hérité de la doctrine théophrastique ${ }^{64}$, ce mode d'évaluation du style est représenté de manière stable dans la doctrine cicéronienne, comme en témoigne le De oratore, où les vertus du style donnent son organisation à l'exposé que Crassus consacre à l'elocutio ${ }^{65}$ :

Quinam igitur dicendi est modus melior, nam de actione post uidero, quam ut Latine, ut plane, ut ornate, ut ad id, quodcumque agetur, apte congruenterque dicamus?

En quoi consiste la meilleure manière de parler - je traiterai plus tard de l'action -, sinon dans la correction, la clarté, l'ornement, l'adaptation et l'adéquation au sujet traité, quel qu'il soit ? (Cic., De orat. III, 37).

Le second ensemble de critères d'évaluation ne concerne plus l'elocutio elle-même, mais la narration ${ }^{66}$ qui, pour être efficace, doit être brève (narratio breuis), claire (narratio dilucida/aperta) et crédible (narratio probabilis/ueri similis). Ces trois vertus de la

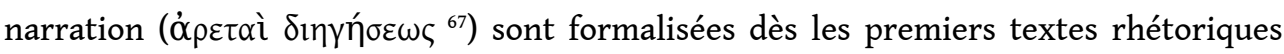
latins qui nous ont été conservés ${ }^{68}$ :

Nunc de narratione ea, quae causae continet expositionem, dicendum uidetur. Oportet igitur eam tres habere res: ut breuis, ut aperta, ut probabilis sit.

Maintenant, je crois qu'il faut parler de cette sorte de narration qui contient l'exposé d'une cause. Il faut donc qu'elle ait trois qualités : qu'elle soit brève, claire, plausible (Cic., De inu. I, 28, trad. G. Achard).

Notre hypothèse est que Sénèque structure les portraits d'orateurs qu'il présente en mêlant indistinctement ces deux structures, vertus du style et vertus de la narration. L'analyse se concentrera sur les quatre déclamateurs qui sont le plus précisément caractérisés dans les préfaces ${ }^{69}$. Deux d'entre eux font partie du tetradeum regroupant, d'après Sénèque, les meilleurs orateurs: Caius Albucius Silus tout d'abord, né vers 60 av.J.-C., et Arellius Fuscus, né en 65 av.J.-C. L'un et l'autre se sont consacrés strictement à la déclamation, Arellius Fuscus n'ayant jamais plaidé, Albucius s'étant détourné $d u$ forum suite à une mésaventure qu'il y avait subie ${ }^{70}$. Les deux autres déclamateurs concernés, qui n'atteignent pas, quant à eux, au premier rang de l'excellence, sont également des orateurs actifs au forum : il s'agit tout d'abord de Quintus Haterius, né en 63 av. J.-C., et de Cassius Seuerus, né en 40 av. J.-C.

Pour décrire ces personnages, Sénèque le Rhéteur met en œuvre quatre modes de caractérisation qui correspondent chacun à une vertu du style ou de la narration. Il examine en premier lieu la cohérence de la pratique stylistique des rhéteurs, reprenant ainsi la vertu de propriété, qui impose à l'orateur d'adapter son style à sa propre personne, et donc de faire preuve d'une certaine stabilité stylistique ${ }^{71}$. Cette vertu de l' apte congruenterque dicere se trouve mise à mal par trois de nos orateurs. Comme on l'a vu, Arellius Fuscus souffre d'une instabilité stylistique très préjudiciable à la qualité de ses déclamations :

Erat explicatio Fusci Arelli splendida quidem sed operosa et implicata, cultus nimis adquisitus, conpositio uerborum mollior quam ut illam tam sanctis fortibusque praeceptis praeparans se animus pati posset; summa inaequalitas orationis, quae modo exilis erat, modo nimia licentia uaga et effusa: principia, argumenta, narrationes aride dicebantur, in descriptionibus extra legem omnibus uerbis dummodo niterent permissa libertas; nihil acre, nihil solidum, nihil horridum; splendida oratio et magis lasciua quam laeta. 
Les développements d'Arellius Fuscus étaient brillants, il est vrai, mais pénibles et embrouillés, l'ornementation trop travaillée, la disposition des mots trop lâche pour plaire à une âme qui se préparait à des préceptes si nobles et si rigoureux. Son style était extrêmement changeant, tantôt sec, tantôt diffus et sans retenue, sous l'effet d'une liberté excessive : l'exorde, la démonstration et les narrations étaient traités de manière sèche, dans les descriptions, s'affranchissant de toute loi, il se permettait librement tous les mots pourvu qu'ils eussent de l'éclat; aucune vigueur, aucun fond, aucune âpreté, c'était un style éclatant et exubérant plutôt qu'abondant (Sen. Rhet., Contr. II praef. 1, trad. H. Bornecque modifiée).

51 Cassius Seuerus souffre d'un défaut proche, puisqu'il parsème ses déclamations d'éléments qui rompent leur unité :

Declamationes eius inaequales erant, sed ea quae eminebant, in quacumque declamatione posuisses, inaequalem eam fecissent.

Ses déclamations étaient inégales, mais ce qui en ressortait aurait suffi à faire paraître inégale n'importe quelle déclamation où on l'aurait introduit (Sen. Rhet., Contr. III praef. 18, trad. H. Bornecque modifiée).

Ce même défaut d'inaequalitas se retrouve chez Caius Albucius Silus qui, plus que tout autre, enfreint la règle de l'apte congruenterque dicere en usant d'un style élevé (splendidissimus) tout en se plaisant à mentionner des réalités basses (res sordidissimae) :

Inaequalitatem in illo mirari licebat. Splendidissimus erat; idem res dicebat omnium sordidissimas - acetum et puleium et tdammam et philerotem † lanternas et spongias : nihil putabat esse quod dici in declamatione non posset.

On pouvait donc légitimement s'étonner des disparités de son propos : il était très brillant et, en même temps, il mentionnait les choses les plus basses; vinaigre, pouliot, daim, rhinocéros, lanternes, éponges, il pensait que l'on pouvait tout mentionner dans une déclamation (Sen. Rhet., Contr. VII praef. 3, trad. H. Bornecque modifiée).

Il transgresse ainsi directement l'exigence cicéronienne d'adaptation du style au sujet :

Quam enim indecorum est, de stillicidiis cum apud unum iudicem dicas, amplissimis uerbis et locis uti communibus, de maiestate populi Romani summisse et subtiliter.

Combien, en effet, il est peu convenable, alors que l'on parle devant un juge unique d'une affaire d'écoulement des eaux, de faire usage de grands mots et de lieux communs, mais de parler avec simplicité et minutie de la majesté du peuple romain (Cic., Orat. 72).

Plus encore, Albucius tend à changer constamment de style, par manque, comme on l'avait vu, de confiance dans ses propres qualités ${ }^{72}$ :

Nulla erat fiducia ingenii sui, et ideo adsidua mutatio ; itaque dum genera dicendi transfert et modo exilis esse uolt nudisque rebus haerere, modo horridus et ualens potius quam cultus, modo breuis et concinnus, modo nimis se attollit, modo nimis se deprimit, ingenio suo inlusit et longe deterius senex dixit quam iuuenis dixerat...

Il n'avait aucune confiance en ses propres moyens: de là ces changements perpétuels; aussi, en passant d'un style à l'autre, en voulant être tantôt sec et s'en tenir à la simple nudité des choses, tantôt rude et énergique plutôt qu'élégant, tantôt bref et précis, en s'élevant parfois trop haut, en redescendant trop bas, il gâta son talent et parla dans sa vieillesse beaucoup plus mal que dans son âge mûr... (Sen. Rhet., Contr. VII praef. 5, trad. H. Bornecque).

55 À cette première grille d'analyse s'en ajoute une deuxième qui oppose la vigueur à la mollesse stylistique. Cette vigueur et cette mollesse tiennent à l'arrangement des mots (compositio) : elles doivent donc s'analyser en rapport avec la vertu de l'ornate telle qu'elle est formalisée par Cicéron. Arellius Fuscus souffre ainsi d'une compositio trop relâchée : 
Erat [...] Fusci Arelli [...] conpositio uerborum mollior quam ut illam tam sanctis fortibusque praeceptis praeparans se animus pati posset.

La disposition des mots était trop lâche chez Arellius Fuscus pour plaire à une âme qui se préparait à des préceptes si nobles et si rigoureux (Sen. Rhet., Contr. II praef. 1, trad. H. Bornecque modifiée).

À l'inverse, Cassius Seuerus déploie un style vigoureux, caractérisé par une compositio énergique :

Conpositio aspera et quae uitaret conclusionem, sententiae uiuae.

Sa disposition des mots était heurtée et évitait les clausules, ses traits étaient vifs (Sen. Rhet., Contr. III. praef. 18, trad. H. Bornecque modifiée). exploite une opposition entre retenue et excès stylistique. En référence aux vertus de l' ornate dicere et de l'apte congruenterque dicere, la question de la retenue stylistique fait intervenir à la fois le rapport entre niveau stylistique et sujet (apte congruenterque dicere) et la maîtrise des outils stylistiques permettant d'embellir le discours (ornate dicere). Cette troisième approche stylistique concerne trois des déclamateurs choisis comme exemples, dont un seul semble capable d'exercer sur son propre style le contrôle scrupuleux qu'attend Sénèque le Rhéteur. Ainsi, Arellius Fuscus soigne trop son style et laisse transparaître les efforts que lui a coûtés la composition dans le résultat final qu'il livre à ses auditeurs ${ }^{73}$. Son oratio est également trop libre. Parce qu'il ne contrôle pas assez rigoureusement le choix de son lexique, Arellius Fuscus en vient en effet à employer un style qui perd toute consistance du fait de sa trop grande libertas ${ }^{74}$ :

summa inaequalitas orationis, quae modo exilis erat, modo nimia licentia uaga et effusa: principia, argumenta, narrationes aride dicebantur, in descriptionibus extra legem omnibus uerbis dummodo niterent permissa libertas; nihil acre, nihil solidum, nihil horridum; splendida oratio et magis lasciua quam laeta.

son style était extrêmement changeant, tantôt sec, tantôt diffus et sans retenue, sous l'effet d'une liberté excessive : l'exorde, la démonstration et les narrations étaient traités de manière sèche, dans les descriptions, s'affranchissant de toute loi, il se permettait librement tous les mots pourvu qu'ils eussent de l'éclat; aucune vigueur, aucun fond, aucune âpreté, c'était un style éclatant et exubérant plutôt qu'abondant (Sen. Rhet., Contr. II praef. 1, trad. H. Bornecque modifiée).

Quintus Haterius, pour sa part, manifeste une incapacité profonde à contrôler son discours, incapacité qui apparaît sous plusieurs formes. Son débit, tout d'abord, est trop rapide, au point d'en devenir un véritable défaut (Tanta erat illi uelocitas orationis ut uitium fieret; Contr. IV praef. 7). Plus grave encore, Haterius est incapable de juger par lui-même de l'ampleur qu'il doit donner à ses développements, à tel point qu'il emploie un affranchi chargé de lui indiquer quand il doit poursuivre son propos et quand il doit le réduire ou l'interrompre :

Regi autem ab ipso non poterat; talioquit libertum habebat cui pareret; sic ibat quomodo ille aut concitauerat eum aut refrenauerat. Iubebat eum ille transire, cum aliquem locum diu dixerat : transibat; insistere iubebat eidem loco : permanebat; iubebat epilogum dicere: dicebat. In sua potestate habebat ingenium, in aliena modum.

Se modérer, il en était lui-même incapable et voilà pourquoi il avait un affranchi auquel il obéissait : l'allure de son discours variait suivant que ce personnage l'avait poussé ou retenu: il lui ordonnait de passer à autre chose, quand il avait traité longtemps une idée: il passait à autre chose; d'insister sur une même idée: il insistait; de prononcer la péroraison: il la prononçait. Il avait la main sur son propre talent, mais en laissait le contrôle à un autre (Sen. Rhet., Contr. IV praef. 8 , trad. H. Bornecque). 
L'image de Caius Gracchus se profile derrière cette anecdote, lui qui se faisait avertir par un esclave muni d'une flûte lorsqu'il laissait sa voix se perdre dans les aigus ou les graves : la note produite par l'esclave lui permettait de reprendre le ton juste ${ }^{75}$. Mais la référence implicite à Caius Gracchus ne permet pas de rendre pleinement compte de la faute régulièrement commise par Haterius, dans la mesure où son défaut ne tient pas à la hauteur de sa voix, mais au contrôle des amplifications qu'il utilise dans ses discours : le problème est, dans son cas, moins physique qu'intellectuel. En sus de cette incapacité à percevoir la mesure par soi-même, Haterius emploie un ornement sans cesse excessif. Incapable de choisir son lexique de façon adéquate et de comprendre que le travail du style doit avoir une limite, il sombre dans le ridicule et devient la risée de tous :

... nemo erat scholasticis nec aptior nec similior, sed dum nihil uult nisi culte, nisi splendide dicere, saepe incidebat in ea quae derisum effugere non possent.

... personne n'était mieux fait pour les rhéteurs et plus semblable à eux ; mais en ne voulant rien dire que d'élégant et de brillant, il tombait souvent sur des expressions qui ne pouvaient échapper aux railleries (Sen. Rhet., Contr. IV praef.10, trad. H. Bornecque modifiée).

Caius Albucius Silus, enfin, souffre de la même tendance que Quintus Haterius, et se laisse aller à une elocutio trop rapide (Dicebat enim citato et effuso cursu ; Contr. VII praef. 2).

61 La dernière caractérisation exploitée par Sénèque repose sur l'opposition entre le superflu et l'utile. Elle reprend à la fois l'exigence de brièveté (breuitas) contenue dans la doctrine des vertus de la narration, et l'exigence de clarté (plane dilucideque dicere) qui régit tant les usages du style que ceux du récit. Car l'usage de démonstrations superflues ou trop complexes, mais aussi de figures excessivement développées ou inopportunes, tend à obscurcir le propos ou à lasser l'auditoire. Arellius Fuscus, à force de détail et de complexité, fait perdre leur clarté à ses démonstrations :

Erat explicatio Fusci Arelli splendida quidem sed operosa et implicata.

Les développements d'Arellius Fuscus étaient brillants, il est vrai, mais pénibles et

embrouillés (Sen. Rhet., Contr. II praef. 1, trad. H. Bornecque modifiée).

De la même manière, Albucius Silus se lance, au beau milieu de ses déclamations, dans des développements philosophiques hors de propos et sur lesquels il n'exerce aucun contrôle puisqu'il les laisse se développer à l'infini :

Illa intempestiua in declamationibus eius philosophia sine modo tunc et sine fine euagabatur.

Cette philosophie, déplacée dans les déclamations, se donnait alors carrière sans mesure et sans fin (Sen. Rhet., Contr. VII praef. 1, trad. H. Bornecque).

De plus, ses argumentations sont toujours développées sans la moindre mesure. C'est que, cherchant à dire tout ce qu'il est possible de dire et non ce qu'il faudrait dire, il fait de chaque partie de sa déclamation une déclamation complète :

Saepe declamante illo ter bucinauit, dum cupit in omni controuersia dicere non quidquid debet dici sed quidquid potest. Argumentabatur moleste magis quam subtiliter : argumenta enim argumentis colligebat, et, quasi nihil esset satis firmum, omnes probationes probationibus aliis confirmabat. Erat et illud in argumentatione uitium, quod quaestionem non tamquam partem controuersiae sed tamquam controuersiam implebat.

Souvent, pendant qu'il déclamait, la trompette sonna trois fois, parce que, dans chaque controverse, il voulait exposer non pas tout ce qui doit, mais tout ce qui peut être dit. Son argumentation était difficile à suivre plus qu'habile; il entassait arguments sur arguments, et, comme s'il ne trouvait rien d'assez solide, il prouvait toutes les preuves par de nouvelles preuves. Il avait encore, dans son 
argumentation, un autre défaut : il développait toutes les questions, non comme des parties d'une controverse, mais comme des controverses (Sen. Rhet., Contr. VII praef. 1, trad. H. Bornecque).

\section{Rhéteur lorsque celui-ci lui demande la raison de son incapacité à déclamer de façon} aussi brillante qu'il plaide (quare in declamationibus impar sibi erat; Contr. II praef. 8). Seuerus développe alors une justification en deux points. S'appuyant sur une théorie générale de l'appropriation, qui fait écho à celle du De officiis de Cicéron ${ }^{77}$, l'orateur se défend tout d'abord en expliquant qu'il est impossible d'exceller dans toutes les activités, et que chacun doit parvenir à identifier, puis à cultiver, celles auxquelles il est le mieux adapté. Puis, Seuerus expose la raison pour laquelle l'activité déclamatoire ne peut convenir aux qualités qui sont les siennes. Le point central tient à la nature de la déclamation, que Seuerus ne parvient pas à considérer comme une activité véritable et tangible :

Indicabo tibi affectum meum: cum in foro dico, aliquid ago; cum declamo, id quod bellissime Censorinus aiebat de his qui honores in municipiis ambitiose peterent, uideor mihi in somniis laborare. Deinde res ipsa diuersa est : totum aliud est pugnare, aliud uentilare.

Je vais te dire ma pensée : lorsque je parle au forum, je fais quelque chose ; lorsque je déclame, il me semble, comme le disait très spirituellement Censorinus de ceux qui briguent les honneurs dans les petites villes, il me semble que je travaille en songe. Ensuite, les conditions sont très différentes: une chose est de combattre, autre chose de faire de l'escrime (Sen. Rhet., Contr. III praef. 12, trad. H. Bornecque modifiée).

La différence tient d'après lui au fait que, contrairement au comportement qu'il parvient à adopter sur le forum, son attention n'est pas concentrée sur un juge ou un adversaire lorsqu'il déclame, mais uniquement sur l'auditoire. Dépourvu de tout enjeu réel, l'exercice lui paraît tout simplement inutile : 
adsueui non auditorem spectare sed iudicem; adsueui non mihi respondere sed aduersario; non minus deuito superuacua dicere quam contraria. In scholastica quid non superuacuum est, cum ipsa superuacua sit?

J'ai coutume de m'adresser non pas à l'auditoire mais au juge, j'ai coutume de répondre non pas à moi-même mais à mon adversaire: je n'évite pas moins les paroles inutiles que celles qui vont contre mon but. Or, dans les exercices d'école, tout n'est-il pas inutile, puisqu'eux-mêmes sont inutiles? (Sen. Rhet., Contr. III praef. 12, trad. H. Bornecque modifiée).

À cette inutilité induite par la nature même de la déclamation, s'ajoute le fait que l'exercice se trouve privé de tout critère d'évaluation stable. Alors que l'éloquence du forum est un combat réel (pugnare), la déclamation n'est qu'un entraînement (uentilare), selon l'opposition déjà utilisée par Cicéron dans le De oratore ${ }^{78}$. En conséquence, les déclamateurs ne se jugent et ne sont jugés que sur des critères invalides, puisque les victoires qu'ils remportent ne sont pas fondées sur un verdict définitif mais sur des appréciations fluctuantes formulées par un public au goût corrompu :

Non est quod oratorem in hac puerili exercitatione spectes. Quid si uelis gubernatorem in piscina aestimare ? [...] Non illi peius dicunt, sed hi corruptius iudicant...

Il n'est pas possible de juger un orateur par cet exercice puéril. C'est comme si l'on voulait essayer un pilote sur un vivier. [...] Ceux-là ne sont pas moins éloquents, mais ceux-ci ont le goût plus mauvais (Sen. Rhet., Contr. III praef.15, trad. H. Bornecque modifiée).

L'efficacité et la capacité de persuasion disparaissent alors totalement du champ de l'évaluation, le jugement formulé sur le déclamateur reposant uniquement sur des critères esthétiques. Dans cette pratique qui trouve en elle-même sa propre fin, le but n'est plus de faire approuver la cause que l'on défend, mais simplement de faire apprécier sa propre virtuosité. La gratuité complète de l'exercice entraîne à sa suite l'emphase, des développements inutiles, et l'inflation d'une éloquence qui n'a plus, désormais, de pierre de touche extérieure à elle-même. Plus rien ne lui permet donc de se contrôler véritablement, ainsi que le souligne Votienus Montanus, dans la préface du livre IX, lorsqu'il reproche aux déclamateurs de ne pas chercher à convaincre mais à plaire :

Qui declamationem parat, scribit non ut uincat sed ut placeat. Omnia itaque lenocinia [ita] conquirit; argumentationes, quia molestae sunt et minimum habent floris, relinquit; sententiis, explicationibus audientis delinire contentus est. Cupit enim se approbare, non causam. Sequitur autem hoc usque in forum declamatores uitium, ut necessaria deserant dum speciosa sectantur.

Celui qui prépare une déclamation écrit non pour remporter la victoire mais pour plaire. Il est donc en quête de toutes les séductions : le développement des preuves, qui est ennuyeux et peu propice aux ornements, il le laisse de côté : il lui suffit de charmer l'auditoire par des traits et des développements. C'est qu'il veut que le succès aille à lui et non à la cause. Ce travers poursuit les déclamateurs jusqu'au forum, où ils abandonnent le nécessaire pour courir après le brillant (Sen. Rhet., Contr. IX praef. 1-2, trad. H. Bornecque modifiée).

On constate alors que les critiques formulées par ces deux personnages à l'égard du genre déclamatoire recoupent précisément les catégories utilisées par Sénèque le Rhéteur pour caractériser les différents orateurs qu'il présente : à la vacuité du propos s'oppose son efficacité, au contrôle des argumentaires un abandon aux développements les plus faciles, à la retenue des effets stylistiques une emphase coupable. Tout en reprenant la théorie des vertus oratoires, la grille de lecture qu'utilise implicitement Sénèque le Rhéteur, lorsqu'il tente de caractériser les différents orateurs et déclamateurs de son temps, recouvre donc également celle qu'emploient Seuerus et 
Montanus lorsqu'ils critiquent la pratique de la déclamation et cherchent à valoriser la pratique oratoire véritable. Les divers personnages que Sénèque passe en revue se trouvent donc inscrits dans un schéma binaire, mais qui ne consiste plus à mettre en balance, comme le faisait le Brutus du Cicéron, deux types bien distincts de qualités. À l'inverse, et selon une approche qui est nouvelle dans la tradition latine, la structuration utilisée par Sénèque oppose globalement qualités et défauts, les qualités étant précisément celles que l'on attendrait d'un orateur véritable, les défauts, d'après les critiques de Seuerus et de Montanus, tenant à la nature de la déclamation. Ainsi le personnage même de Seuerus, qui, dans les oppositions que nous avons dressées, représente fréquemment un modèle positif, est-il orateur avant d'être déclamateur (Contr. III praef. 7). Ses qualités tiennent donc à sa pratique oratoire efficace, quand ses défauts découlent précisément de la gratuité qu'il impute à la déclamation. Ainsi, Sénèque oppose entre eux les grands déclamateurs selon qu'ils manifestent ou non les défauts consubstantiels au genre qu'ils pratiquent tous. Par cette mise en écho des critiques explicitement formulées dans l'ouvrage et des caractérisations qu'il nous livre, on perçoit clairement que les Controuersiae présentent une réflexion rhétorique construite qui dépasse largement le simple parcours mémoriel auquel Sénèque prétendait tout d'abord se livrer.

\section{Conclusion}

71 En critiquant le rhéteur Bassus, au livre $\mathrm{X}$ de son ouvrage, Sénèque le Rhéteur souligne l'indécence qu'il y a, pour un rhéteur, à imiter les pratiques d'un forum dont il ignore tout :

Nihil est indecentius quam ubi scholasticus forum quod non nouit imitatur.

Rien n'est plus inconvenant qu'un rhéteur qui imite le forum auquel il ne connaît rien (Sen. Rhet., Contr. X praef. 12).

Mais la description qu'il nous livre des orateurs et des rhéteurs de son temps montre également que rien n'est plus condamnable qu'un rhéteur qui, en profitant pleinement des libertés que lui offre le genre déclamatoire, a oublié les contraintes qui donnaient à l'éloquence du forum toutes ses qualités. Par ce biais détourné et contre toute attente, Sénèque le Rhéteur inscrit donc son propos dans l'histoire, et fait des caractéristiques des différents déclamateurs le produit d'une modification historique et politique qui a cantonné l'éloquence au domaine de l'école.

Tout en ménageant une place aux évolutions et aux goûts du temps, la grille de lecture des caractéristiques oratoires que Sénèque met implicitement en place valorise pour l'essentiel des qualités propres à l'éloquence du forum et critique des défauts liés de façon nécessaire à la pratique déclamatoire. Cette analyse entraîne donc avec elle une lecture selon laquelle le bon orateur est celui qui a su ne pas s'abandonner aux libertés de l'époque et qui, selon Sénèque, ne s'est pas laissé aller à la décadence. On comprend alors que cette architecture rhétorique et stylistique ne trouve son sens et sa pertinence que dans le cadre critique et historique mis en place par l'auteur: la compréhension des caractéristiques, qualités comme défauts, des différents déclamateurs n'est possible que si le lecteur prend en compte l'évolution des pratiques et, plus encore, la disparition de l'éloquence efficace. Le projet mémoriel de Sénèque, qui vise à préserver les exemples anciens valides - quoique pour certains déjà corrompus -, vient ainsi au secours d'une entreprise rhétorique plus critique que 
descriptive, et qui doit également s'entendre comme une volonté de réformer les pratiques oratoires du temps. Perspective historique et perspective rhétorique ne peuvent plus, dès lors, être dissociées.

\section{BIBLIOGRAPHIE}

BARNES T.D. 1986, «The Significance of Tacitus' Dialogus de Oratoribus », HSPh 90, p. 225-244.

BONNER S.F. 1949, Roman Declamation in the Late Republic and Early Empire, Liverpool - Berkeley Los Angeles.

BORNECQUE H. 1967, Les déclamations et les déclamateurs d'après Sénèque le Père, Hildesheim [reprod. photomécanique de l'éd. de : Lille, 1902].

CALBOLI MONTEFUSCO L. 1988, Exordium, narratio, epilogus: studi sulla teoria retorica greca e romana delle parti del discorso, Bologne.

Calboli Montefusco L. 2003, «Ductus and Color: The Right Way to Compose a Suitable Speech », Rhetorica 21, p. 113-131.

CHIRON P. 2001, Un rhéteur méconnu : Démétrios (Ps.-Démétrios de Phalère). Essai sur les mutations de la théorie du style à l'époque hellénistique, Textes et traditions 2, Paris.

DAVID J.-M. 1980, «Eloquentia popularis et conduites symboliques des orateurs de la fin de la République : problèmes d'efficacité », QS 12, p. 171-211.

DelPeyroux M.-F. 2003, « Sénèque le Père et la décadence de la rhétorique », in B. Bakhouche (éd.), L'ancienneté chez les Anciens. II, La vieillesse dans les sociétés antiques : la Grèce et Rome, Montpellier, p. 629-651.

Douglas A.E. 1966, « Oratorum Aetates », AJPh 87, p. 290-306.

Ducrot O. 1984, Le dire et le dit, Propositions, Paris.

FAIRWEATHER J. 1981, Seneca the Elder, Cambridge Classical Studies, Cambridge.

FANTHAM E. 1978a, «Imitation and Decline: Rhetorical Theory and Practice in the First Century After Christ », CPh 73, p. 102-116.

FANTHAM E. 1978b, «Imitation and Evolution: The Discussion of Rhetorical Imitation in Cicero De Oratore 2. 87-97 and Some Related Problems of Ciceronian Theory », CPh 73, p. 1-16.

FANTHAM E. 1979, « On the Use of Genus-Terminology in Cicero's Rhetorical Works », Hermes 107, p. 441-459.

FORTENBAUGH W.W. (éd.) 2005, Theophrastus of Eresus: Sources for His Life, Writings, Thought and Influence, Commentary Volume 8, Sources on Rhetoric and Poetics (Texts 666-713), Philosophia antiqua 97, Leyde.

GotofF H.C. 1984, « Review: Seneca the Elder by Fairweather, Janet », CPh 79, p. 166-167. 
GUÉRIN C. 2009, Persona : l'élaboration d'une notion rhétorique au I ${ }^{\text {er }}$ siècle av. J.-C. I, Antécédents grecs et première rhétorique latine, Textes et traditions 18 , Paris.

GUÉRIN C., à paraître a, « La tablette et la torche : mémoire, composition et performance oratoire dans la rhétorique romaine ", in C. Jacob (éd.), Les lieux de savoir. II, Les mains de l'intellect, Paris.

GUÉRIN C., à paraître b, «Oratorum bonorum duo genera sunt : la redéfinition de l'excellence stylistique et ses conséquences dans le Brutus de Cicéron », in S. Aubert (éd.), Prosopographie et rhétorique : le Brutus, archétype de l'histoire culturelle, Louvain.

INNES D. 1984, «Theophrastus and the Theory of Style ", in W.W. Fortenbaugh, P.M. Huby, A.A. Long (éds), Theophrastus of Eresus: On His Life and Work, Rutgers University Studies in Classical Humanities 2, New Brunswick (N.J.) - Oxford, p. 251-267.

KENNEDY G.A. 1972, The Art of Rhetoric in the Roman World, 300 B.C-A.D. 300, Princeton (N.J.).

LEVENE D.S. 2004, « Tacitus’ Dialogus As Literary History », TAPhA 134, p. 157-200.

LÉVY C. 2006, «La notion de color dans la rhétorique latine : Cicéron, Sénèque le Rhéteur, Quintilien ", in A. Rouveret, V. Naas, S. Dubel (éds), Couleurs et matières dans l'Antiquité : textes, techniques et pratiques, Études de littérature ancienne 17, Paris, p. 185-199.

Mitchell T. 1979, Cicero: The Ascending Years, New Haven - Londres.

MOMIGLIANO A. 1991, La naissance de la biographie en Grèce ancienne, Strasbourg.

Sochatoff A. 1939, « Basic Rhetorical Theories of the Elder Seneca », CJ 34, p. 345-354.

STEEL C.E.W. 2002-2003, « Cicero's Brutus: The End of Oratory and the Beginning of History? », BICS

46, p. 194-211.

SUMNER G.V. 1973, The Orators in Cicero's Brutus: Prosopography and Chronology, Phoenix. Suppl. vol. 11, Toronto.

SusSmAN L.A. 1971, «The Artistic Unity of the Elder Seneca's First Preface and the Controuersiae as a Whole », AJPh 92, p. 285-291.

SuSSMAN L.A. 1978, The Elder Seneca, Mnemosyne. Suppl. 51, Leyde.

SYME R. 1938, « The Allegiance of Labienus », JRS 28, p. 113-125.

\section{NOTES}

1. Sur la datation des écrits de Sénèque le Rhéteur, cf. Fairweather 1981, p. 15.

2. Sur l'évolution de la notion rhétorique de color et le sens qu'elle en vient à prendre chez Sénèque le Rhéteur, cf. Calboli Montefusco 2003 et Lévy 2006.

3. Sur la déclamation en tant que genre, cf. Bonner 1949 ; Kennedy 1972, p. 312-337.

4. Sur les différentes mutilations et modifications subies par l'ouvrage, cf. Bornecque 1967, p. 32-38; Fairweather 1981, p. 34-35.

5. Une liste complète des déclamateurs cités, accompagnée de notices biographiques, est fournie par Bornecque 1967, p. 143-201.

6. C'est du moins le cas dans les parties intégralement conservées, les livres transmis sous la forme d'excerpta ayant fait disparaitre ces indications.

7. Sur la notion rhétorique de persona, cf. Guérin 2009.

8. Sussman 1978, p. 95-96.

9. Sussman 1978, p. 94. 
10. Fairweather 1981, p. 51-55.

11. Cf. Fairweather 1981, p. 51 : «Seneca's character sketches do not lend themselves to systematic analysis or classification. » Voir également Sussman 1978, p. 95.

12. Sur la mise en œuvre de cette rhétorique descriptive dans le Brutus, cf. Guérin, à paraître b.

13. Sussman 1978, p. 97.

14. Cf. Fairweather 1981 , p. 56 et p. 67 , qui réduit le Brutus à un traité polémique contre les Atticistes, où Cicéron chercherait à faire la théorie de l'orateur idéal.

15. Cf. Fairweather 1981, p. 59-67, qui affirme: «It is with the biographical tradition that the elder Seneca's work has most affinity » (p. 59).

16. Cf. Fairweather 1981, p. 55 : «most [parts of the work] are descriptive of a particular declaimer's approach to a particular thema, rather than being cast in the form of didactic generalizations. Hence, before we can compare his views with those of, for example, Cicero and Quintilian, we have to impose a framework on his criticism which bears no relation to the way in which it was originally set out. For Seneca the Elder is unusual among ancient critics in feeling no desire to arrange his criticism according to the rigid divisions of oratory prescribed in the rhetorical handbooks. » On se reportera, à propos de cette méthode, aux remarques de Gotoff 1984.

17. Fairweather 1981, p. 56 : «There are certain recurring themes to be found in Seneca's penportrait, but they have nothing to do with classifications set out in rhetorical handbooks. » On remarquera d'emblée qu'une telle unification de la doctrine ( rhetorical handbooks ») tend à créer un objet rhétorique coupé de toute réalité historique et, par conséquent, entièrement fictif.

18. Sussman 1971.

19. Cf. la synthèse proposée par Sochatoff 1939.

20. Brut. 20.

21. Dans le Brutus, le personnage même d'Atticus qualifie le propos cicéronien d'historia (Brut. 292). Sur la structuration du Brutus et les sources qui l'ont inspirée, cf. Sumner 1973, p. 2-4 et 151-176 ainsi que la bibliographie fournie.

22. Cf. les exigences formulées par Cicéron en De orat. II, 63-64.

23. La mention de cette demande reprend bien sûr une convention littéraire d'usage courant dans les préfaces d'œuvres en prose, en particulier dans celles des textes rhétoriques. Cf. sur ce point Sussman 1978, p.51-52, qui analyse la forme épistolaire que prennent les préfaces de Sénèque, et Fairweather 1981, p. 27.

24. Contr. X praef. 16.

25. Ainsi, le déclamateur Musa apprécié par Mela : cf. Contr. X praef. 9.

26. L'échange entre les personnages d'Atticus et de Cicéron, en Brut. 244, montre bien le souci d'exhaustivité qui anime le projet historique cicéronien. Comme le lui reproche Atticus, Cicéron est en effet amené à recenser des individus qui, d'après ses interlocuteurs, ne le méritent pas : l'enjeu cependant n'est pas de recenser les bons orateurs de Rome, mais tous ceux qui ont tenté de prendre la parole en public. Cette exhaustivité permet alors de démontrer qu'il est peu d'orateurs véritablement dignes de mémoire: Volo autem hoc perspici, omnibus conquisitis, qui in multitudine dicere ausi sint, memoria quidem dignos perpaucos, uerum qui omnino nomen habuerint, non ita multos fuisse (Brut. 244).

27. Sur cette prétention et sa valeur conventionnelle, cf. Fairweather 1981, p. 37-39. Sur la thématique de la mémoire dans les Controuersiae et ses implications rhétoriques, cf. Guérin, à paraître a.

28. L'affirmation de Sénèque le Rhéteur est néanmoins plusieurs fois contredite par son propre texte. J. Fairweather souligne ainsi que des sources écrites sont mentionnées à plusieurs reprises dans les Controuersiae : cf. Fairweather 1981, p. 40-41. 
29. Cf. Contr. I praef. 20. Sénèque le Rhéteur adopte donc la posture qui était celle de Cicéron dans le prologue du livre II du De oratore (§ 1-12), où l'Arpinate entendait défendre la mémoire de ses maîtres et modèles, Licinius Crassus et Marcus Antonius.

30. Contr. I praef. 10.

31. Sussman 1971.

32. Cf. les remarques de Fairweather 1981, p. 30.

33. Sénèque souligne que la déclamation pratiquée à son époque est apparue après sa naissance, et qu'il peut donc traiter sans difficulté d'un genre dont il a connu le développement depuis son origine : nam et studium ipsum nuper celebrari coepit : ideo facile est mihi ab incunabulis nosse rem post me natam (Contr. I praef. 12).

34. Cf., pour Cassius Seuerus, Contr. III praef. 3 et, pour Albucius, Contr. VII praef. 5.

35. Sen. Rhet., Contr. X praef. 4-5.

36. Sur la dimension sérielle de la biographie, cf. les remarques de Momigliano 1991, p. 26.

37. Tout en insistant sur la proximité des textes de Sénèque le Rhéteur et des biographies qui nous ont été conservées, Fairweather 1981, p. 66-67, souligne que «there is no evidence [...] that any author wrote character-sketches of the rhetoricians of the late Republic resembling those in Seneca's prefaces. [...] So, in the absence of any obvious precursor to Seneca the Elder in his mode of pen-portraiture, we have to treat him as an individualist and innovator, which maybe is indeed what in some respects he was."

38. Le projet consistant à répartir les genres d'orateurs par époque (oratorum genera distinguere aetatibus, Brut. 74) aboutit logiquement à identifier une figure dominante pour chaque aetas. Sur ce projet, cf. la synthèse de Douglas 1966.

39. Sur le sens que prend le terme genus dans ce contexte, cf. Fantham 1979, p. 447-449.

40. Cf. Steel 2002-2003, p. 209.

41. Cf. en particulier Brut.201. Sur l'innovation rhétorique que présente ce mode de structuration et ses conséquences sur l'appréciation de l'excellence oratoire, cf. Guérin, à paraître b.

42. Contr. I praef. 12.

43. Ainsi, la modification des divisions traditionnellement utilisées dans les déclamations, qui tendent à devenir plus complexes avec le temps (Contr. I, 1, 13-14).

44. Contr. II praef. 5.

45. Bornecque 1967, p. 138.

46. Sur cette thématique du déclin et son traitement par Sénèque le Rhéteur, cf. Kennedy 1972, p. 446-464 ; Fantham 1978a, p. 111-116 ; Fairweather 1981, p.132-148; Barnes 1986; Delpeyroux 2003.

47. Sur ce personnage, on se reportera à Syme 1938.

48. Tacite, Dialogus de oratoribus 36-41. Cf. Levene 2004, p. 189.

49. Tacite, Dialogus de oratoribus 18. Cf. Levene 2004, p. 174-175.

50. Le programme de formation proposé par Antoine impose en premier lieu à l'apprenti le choix d'un modèle qui lui soit adapté, puis une imitation raisonnée lui permettant d'atteindre une véritable ressemblance (De orat. II, 90-98). Cf. sur ce point Fantham 1978b.

51. La règle énoncée par Sénèque apparaît comme une reformulation pratique du principe théorique énoncé dans le De inuentione par Cicéron, au milieu des années 80 av. J.-C., où l'auteur justifiait la qualité de son ouvrage en insistant sur le nombre de sources qu'il avait pu employer et sur la sélection qu'il y avait effectuée : cf. De inu. II, 4-5.

52. E. Fantham voit dans cette analyse de Sénèque une résurgence de la doctrine platonicienne de l'imitation. Cf. Fantham 1978a, p. 112.

53. L'exigence de diversification des modèles, qui s'éloigne radicalement de la doctrine cicéronienne, se retrouve également chez Quintilien : cf. en particulier Inst. orat. X, 2, 24 et Fantham 1978a, p. 107-108. 
54. De orat. II, 90-92.

55. Ainsi, le rapport de Cicéron à ses modèles, Antoine et Crassus, comporte une dimension politique indéniable (cf. Mitchell 1979, p.42-51). Plus généralement, la reproduction du comportement oratoire d'un modèle vaut comme une reprise de ses choix politiques : on sait que la reproduction de l'actio propre aux Gracques valait comme une proclamation d'orientations populares (cf. David 1980).

56. Sur ce processus continu de dégradation, cf. Contr. I praef. 6-7.

57. Sur cette structure rhétorique, cf. Innes 1984 ; Chiron 2001, p. 146-154.

58. Ces types sont appelés genera ou figurae par l'Auctor: cf. Rhet. Her. IV, $11 \mathrm{sq}$.

59. Marullus (Contr. I praef. 22 : Marullus, homo satis aridus... exilitas orationis suae), Arellius Fuscus (Contr. II praef. 1 : narrationes aride dicebantur), Buteo, (Contr. VII, 5, 15 : aridus et exucus declamator), Aemilianus (Contr. X, 5, 35 : Aemilianus quidam Graecus rhetor... ex arido fatuus) auxquels on peut ajouter un rhéteur anonyme qui tient école avec Sparsus (Contr. X praef. 11 : declamator subtilis sed aridus).

60. Arellius Fuscus (Contr. II praef.1: explicatio splendida, splendida oratio), Quintus Haterius (Contr. IV praef. 7 : copia), Caius Albucius Silus (Contr. VII praef. 2 : splendor orationis quantus nescio an in ullo alio fuerit).

61. Cf. en particulier Brut. 86-89, 194-198, 201-203, 317. Sur ces passages, cf. Guérin, à paraître b.

62. Rhet. Her. IV, 14-16.

63. Cf. en particulier la description de la figura sufflata, qui souligne clairement que le défaut consiste à employer de manière excessive ce qui fait la particularité même du style positivement défini : item grauis oratio saepe inperitis uidetur ea, quae turget et inflata est, cum aut nouis aut priscis uerbis aut duriter aliunde translatis aut grauioribus quam res postulat (Rhet. Her. IV, 15).

64. Cf. Innes 1984 ; Chiron 2001, p. 146-154; Fortenbaugh (éd.) 2005, p. 267.

65. L'Orator de Cicéron présentera une formulation identique, cf. Orat. 79.

66. Sur la confusion progressive des vertus du style et des vertus de la narration dans la tradition rhétorique, cf. Calboli Montefusco 1988 ; Chiron 2001, p. 139, n. 264.

67. La théorisation des $\alpha \rho \varepsilon \tau \alpha \grave{~} \delta ı \gamma \eta ́ \sigma \varepsilon \omega \varsigma$ apparaît pour la première fois dans la Rhétorique à Alexandre du Ps.-Aristote, Cf. Rhet. ad Alex. 1438a 19-21.

68. Cf. également Rhet. Her. I, 14 ; De orat. II, 80-83 ; Orat. 124 ; Part. orat. 31-32 ; Top. 97 ; Inst. orat. IV, 2, 31-62.

69. Ces déclamateurs sont ceux dont la pratique est la plus précisément décrite dans les Controuersiae, les informations proprement biographiques les concernant tenant une place moins centrale dans le texte. On peut opposer le cas extrême de Fuscus (cf. Contr. II praef. 1), qui n'est caractérisé qu'au moyen de sa pratique oratoire, et de Porcius Latro (que nous n'avons pas retenu dans notre analyse), dont le portrait est presque entièrement biographique, au détriment de la caractérisation stylistique (cf. Contr. I praef. 13-24).

70. Contr. VII praef. 6-7.

71. Sur le fondement cicéronien de cette exigence, cf. Orat. 70-74. Contrairement à Fairweather 1981 p. 56-57, qui analyse la thématique de l'inaequalitas comme biographique et non rhétorique, nous considérons que l'inaequalitas des rhéteurs doit être rapprochée de leur persona stylistique, et que les développements que Sénèque lui consacre doivent par conséquent se comprendre comme une critique proprement rhétorique.

72. Suétone fusionne les deux critiques (mélange de hauteur et de trivialité et changements stylistiques) dans la biographie d'Albucius qui nous a été transmise : Sed ex eo clarus propria auditoria instituit, solitus proposita controuersia sedens incipere et calore demum prouectus consurgere ac perorare, declamare autem genere uario, modo splendide atque adornate tum ne usque quaque scholasticus existimaretur circumcise ac sordide et tantum non triuialibus uerbis (De gram. rhet. XXX, 3).

73. Contr. II praef. 1. 
74. Un reproche presque identique est formulé à l'égard d'Arellius Fuscus en Suas. II, 23 : Quarum nimius cultus et fracta conpositio poterit uos offendere cum ad meam aetatem ueneritis; interim <non> dubito quin nunc uos ipsa quae offensura sunt uitia delectent.

75. De orat. III, 225 ; Inst. orat. I, 10, 27 ; Val. Max., VIII, 10.

76. La critique s'est souvent posé la question de savoir dans quelle mesure Sénèque le Rhéteur pouvait partager les points de vue qu'il rapportait dans ses préfaces. J. Fairweather considère que Sénèque adopte uniformément une posture non critique à l'égard de la déclamation. Mais rien ne permet d'étayer une telle affirmation, qui néglige d'ailleurs de prendre en compte la polyphonie énonciative située au fondement des Controuersiae. Si elles ne sont pas explicitement assumées par Sénèque, les critiques formulées par Seuerus et Montanus ne sont pas pour autant affaiblies ou contestées par l'auteur : elles peuvent par conséquent être pleinement intégrées au propos de l'ouvrage. Sur la notion de polyphonie énonciative, cf. Ducrot 1984, p. 192 sq.

77. Cf. en particulier De off. I, 110-114 et 117-121.

78. Cf. De orat. II, 84.

\section{RÉSUMÉS}

L'objet de cet article est d'analyser les structures rhétoriques d'interprétation qui sont mises en place par Sénèque le Rhéteur pour rendre intelligibles les caractéristiques des déclamateurs et présenter leurs personae respectives dans les préfaces des Controversiae. Il s'interroge tout d'abord sur le rapport général qu'entretient le texte à l'histoire, à la biographie et à la rhétorique, pour examiner ensuite la manière dont Sénèque le Rhéteur modifie les outils d'analyse propres à la rhétorique descriptive dont il est l'héritier afin de les adapter à son objet et de leur donner une valeur historique nouvelle. Le projet mémoriel de Sénèque, qui vise à préserver les exemples anciens valides - quoique pour certains déjà corrompus -, vient ainsi au secours d'une entreprise rhétorique plus critique que descriptive, et qui doit également s'entendre comme une volonté de réformer les pratiques oratoires du temps. 


\section{INDEX}

Mots-clés : biographie, colores, controverse, critique, déclamation, dicendi uirtutes, didactique (fonction didactique de la littérature), dispositio, diuisiones, elocutio, éloge, enseignement à Rome, imitation (mimèsis), littérature antiquaire, norme rhétorique, orateurs romains, plagiat, portrait, rhétorique romaine, tradition rhétorique, sententiae, vie (genre littéraire), théorie

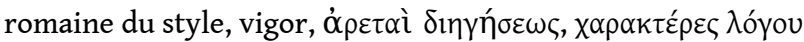

Keywords : biography, colores, controversia, criticism, declamation, dicendi uirtutes, didactics (didactic function in literature), dispositio, diuisiones, elocutio, praise, Roman education, imitatio, antiquarian literature, rhetorical norms, Roman orators, plagiarism, portrait, Roman rhetoric, rhetoric tradition, sententiae, life (literary genre), Roman theories on style, vigor,

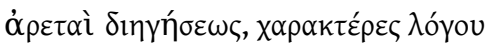

nomsmotscles Aemilianus, Albucius Silus, Arellius Fuscus, Atticus, Buteo, Cassius Severus, Cestius Pius, Cicéron, Fulvius Sparsus, Gallion, Lucius Asprenus, Lucius Magius, Marullus, Papirius Fabianus, Porcius Latro, Pseudo-Aristote, Quintilien, Quintus Haterius, Sénèque le Rhéteur, Suétone, Titus Labienus, Votienus Montanus

\section{AUTEURS}

\section{CHARLES GUÉRIN}

Université Paul-Valéry Montpellier III 